\title{
Generation and Molecular Characterization of Transient tet1/2/3 Zebrafish Knockouts
}

\section{Samuel E. Ross and Ozren Bogdanovic}

\begin{abstract}
5-methylcytosine $(5 \mathrm{mC})$ is a gene-regulatory mark associated with transcriptional repression. $5 \mathrm{mC}$ can be erased through the catalytic action of Ten-eleven translocation (TET) methylcytosine dioxygenases (TET1, TET2, TET3), which oxidize $5 \mathrm{mC}$ resulting in its removal from the genome. In vertebrates, TET enzymes facilitate DNA demethylation of regulatory regions linked to genes involved in developmental processes. Consequently, TET ablation leads to severe morphological defects and developmental arrest. Here we describe a system that can facilitate the study of relationships between TET enzymes, $5 \mathrm{mC}$, and embryo development. We provide detailed descriptions for the generation of F0 zebrafish tet $1 / 2 / 3$ knockouts using CRISPR/Cas 9 technology and elaborate on the strategies to assess the impact of TET loss by reduced representation bisulfite sequencing (RRBS).
\end{abstract}

Key words Reduced representation bisulfite sequencing (RRBS), DNA methylation, TET proteins, Zebrafish, CRISPR/Cas9, DNA demethylation, Embryogenesis

\section{Introduction}

5 -methylcytosine $(5 \mathrm{mC})$ is a chemical modification of vertebrate genomes frequently associated with transcriptional repression [1]. $5 \mathrm{mC}$ is generated by the transfer of a methyl group from $S$-adenosylmethionine (SAM) to the fifth carbon of the cytosine pyrimidine ring, and this reaction is catalyzed by DNA methyltransferases (DNMTs). Vertebrate embryogenesis is characterized by extensive $5 \mathrm{mC}$ dynamics [2]. $5 \mathrm{mC}$ is deposited de novo by DNMT3a and DNMT3b and is maintained through DNA replication by DNMTI $[3,4] .5 \mathrm{mC}$ can be passively lost by exclusion of DNMTl activity from the nucleus during DNA replication, or

The original version of this chapter was revised. The correction to this chapter is available at https://doi.org/ 10.1007/978-1-0716-1294-1_18

Data Availability: RRBS data were deposited at the Gene Expression Omnibus under the record: GSE149424.

Ozren Bogdanovic and Michiel Vermeulen (eds.), TET Proteins and DNA Demethylation: Methods and Protocols, Methods in Molecular Biology, vol. 2272, https://doi.org/10.1007/978-1-0716-1294-1_17,

(c) The Author(s) 2021, Corrected Publication 2021 
actively by a family of enzymes known as Ten-eleven translocation (TET) methylcytosine dioxygenases [5, 6]. Many vertebrates, including mammals and zebrafish, encode three TET protein copies (TET1, TET2, TET3). TET enzymes catalyze the iterative oxidation of $5 \mathrm{mC}$ to 5 -hydroxymethylcytosine ( $5 \mathrm{hmC}), 5$-formylcytosine (5fC), and 5-carboxylcytosine $(5 \mathrm{caC})$ [5-7]. In vitro studies have demonstrated that $5 \mathrm{fC}$ and $5 \mathrm{caC}$ can be removed from the DNA and replaced with unmethylated cytosines by thymine DNA glycosylase (TDG) and base-excision repair machinery (BER) $[8,9]$. TET knockout animals have revealed both redundant and specialized roles for each TET protein, with animals deficient for all three TET proteins exhibiting most severe developmental phenotypes [10]. The majority of triple TET knockout (TKO) mice failed to survive past gastrulation, due to hyperactive Nodal signaling caused by reduced expression of Leftyl and Lefty 2 genes [11]. Similarly, the majority of triple tet zebrafish morphants (tet1/tet2/tet3) displayed embryonic lethality; however, a minor percentage of embryos that survived gastrulation exhibited impaired head structures, shortened axes, microphthalmia, and reduced pigmentation [12]. TALEN-induced zebrafish tet TKOs were characterized by brain abnormalities, smaller eyes, altered pigmentation, trunk curvature and did not survive beyond the larval period [13]. These animal studies indicate that TET proteins are dispensable for pluripotency but are required for organ development and body plan formation.

Whole genome bisulfite sequencing (WGBS) of mouse, zebrafish, and frog embryos revealed TET-mediated demethylation of developmental enhancers during the vertebrate phylotypic period [12]. Phylotypic period is the most conserved phase of vertebrate development that is associated with hox cluster expression and organogenesis [14]. WGBS analysis of tet $1 / 2 / 3$ zebrafish morphants also unraveled that the loss of Tet proteins reduces extent of demethylation, resulting in hypermethylation of these regions [12]. This suggests that the activation of key developmental pathways could be driven by active $5 \mathrm{mC}$ demethylation. These differentially methylated regions (DMRs), which normally become hypomethylated during the phylotypic period, will from here on be referred to as phylo-DMRs.

Here we present a system that can facilitate the studies of TET-mediated $5 \mathrm{mC}$ demethylation during zebrafish embryogenesis. We employ CRISPR/Cas9 technology [15] to produce tet1/2/ $3 \mathrm{KO}$ F0 zebrafish, and reduced representation bisulfite sequencing (RRBS) to measure changes in $5 \mathrm{mC}$ levels [16]. Purified Cas9 nuclease and guide RNAs (gRNA) against all three tet genes are co-injected into single-cell zebrafish zygotes, and DNA and RNA are harvested at $24 \mathrm{~h}$ post fertilization (hpf). The RNA is used for qPCR analyses, whereas the DNA is used for both amplicon sequencing, to validate knockout efficiency, and for RRBS library construction. This system allows for rapid and cost-efficient generation of data, overcoming specificity issues associated with 
morpholino-based approaches $[17,18]$ as well as time, labor, and viability issues associated with generating stable tet $1 / 2 / 3 \mathrm{KO}$ animals. This protocol can be applied to test any gene or gene combination for their involvement in the regulation of DNA methylation.

RRBS is a DNA methylation analysis technique that can be used for assessment of $5 \mathrm{mC}$ levels in CG-dense regions, such as promoters and certain enhancers (i.e., phylo-DMRs), thereby reducing the total sequencing depth required. RRBS is based on restriction enzyme digestion, most commonly MspI, which allows for the tailoring of libraries to specific genomic contexts. Like WGBS, the generation of RRBS libraries entails end repair, adapter ligation, sodium bisulfite conversion, and PCR amplification. Sodium bisulfite promotes the deamination of unmethylated cytosines (C), $5 \mathrm{fC}$, and $5 \mathrm{caC}$ to uracil while $5 \mathrm{mC}$ and $5 \mathrm{hmC}$ do not undergo this transition $[19,20]$. After PCR amplification, this results in $\mathrm{C}, 5 \mathrm{fC}$, and $5 \mathrm{caC}$ being replaced with thymines, and $5 \mathrm{mC}$ and $5 \mathrm{hmC}$ remaining as cytosines. The data generated by RRBS are processed and analyzed using bioinformatics pipelines comparable to those used for WGBS data.

\section{Materials}

\subsection{Zebrafish Husbandry}

\subsection{Equipment}

Zebrafish maintenance and breeding were conducted under full animal use and care guidelines with approval by the Garvan Institute of Medical Research animal care and use committee. The zebrafish used were wild type (Tübingen/AB) and were raised under standard conditions at $28^{\circ} \mathrm{C}$.

1. PCR thermocycler.

2. Real-time PCR thermocycler such as the LightCycler 480 (Roche).

3. DynaMag 96-side Magnetic rack or equivalent.

4. Qubit fluorometer.

5. Microcentrifuge.

6. NanoDrop spectrophotometer.

7. Picospritzer III-intracellular microinjection dispense system.

8. P-87 stutter instrument or equivalent.

9. Stage microruler.

10. Agilent 4200 TapeStation or equivalent.

11. Illumina HiSeq $\mathrm{X}$ sequencing platform and corresponding reagents or equivalent.

1. $0.2 \mathrm{ml}$ PCR tubes.

2. $1.5 \mathrm{ml}$ nuclease-free tubes. 
3. Qubit Assay Tubes.

4. Petri Dishes $90 \mathrm{~mm} \times 15 \mathrm{~mm}$.

5. Petri Dishes $150 \mathrm{~mm} \times 15 \mathrm{~mm}$.

6. Z-MOLDS ZEBRAFISH MI/TRANSPLANT KIT (Coherent Scientific).

7. Scalpels.

8. Thin wall glass capillaries $(0.75 \mathrm{~mm}$, od: $1 \mathrm{~mm})$ (World Precision Instruments).

9. Pasteur pipettes.

10. Tapestation D1000 ScreenTape and reagents (Agilent Technologies).

11. Microloader pipette tips (Eppendorf).

\subsection{Reagents}

1. Nuclease-free water.

2. $50 \mathrm{mM} \mathrm{MgCl}$.

3. $10 \mathrm{mM}$ DNTPs.

4. $1 \mathrm{mM}$ DNTPs.

5. Taq DNA Polymerase, recombinant (Invitrogen).

6. PCR buffer $(10 \times): 200 \mathrm{mM}$ Tris-HCl, $\mathrm{pH} 8.4,500 \mathrm{mM} \mathrm{KCl}$.

7. AMPure XP (Agencourt).

8. Ethanol (molecular biology grade).

9. AMPLISCRIBE Tм T7-FLASH Tм TRANSCRIPTION KIT (Lucigen).

10. Qubit ${ }^{\text {TM }}$ RNA BR Assay Kit.

11. Qubit ${ }^{\text {TM }}$ DNA HS Assay Kit.

12. Agarose powder.

13. Phenol Red.

14. EnGene CAS9 NLS, S Pyogenes (NEB).

15. E3 solution: $5 \mathrm{mM} \mathrm{NaCl}, 0.17 \mathrm{mM} \mathrm{KCl}, 0.33 \mathrm{mM} \mathrm{CaCl}_{2}$, $0.33 \mathrm{mM} \mathrm{MgSO}_{4}$.

16. DNeasy Blood and Tissue Kit (Qiagen) or equivalent.

17. TRIsure (Bioline).

18. Phenol:Chloroform:Isoamyl Alcohol 25:24:1.

19. Chloroform.

20. SensiFAST ${ }^{\text {TM }}$ cDNA Synthesis Kit (Bioline).

21. KAPA SYBR FAST qPCR Master Mix $(2 \times)$ Universal (KAPA Biosystems).

22. MspI restriction endonuclease $[20 \mathrm{U} / \mu \mathrm{l}](\mathrm{NEB})$.

23 . Unmethylated $\lambda$ phage DNA (Promega).

24. $10 \mathrm{mM}$ dATP. 
25. Klenow Fragment $\left(3^{\prime}-5^{\prime}\right.$ exo- $)$ (NEB).

26. NEXTflex bisulfite-seq barcodes and NEXTflex universal primer (Bioo Scientific).

27. EZ DNA Methylation-Gold Kit (Zymo Research).

28. KAPA HiFi HotStart Uracil + ReadyMix (Kapa Biosystems).

29. Library Quantification Kit-Illumina/Universal (KAPA Biosystems).

\section{Methods}

\subsection{CRISPR/Cas9 Guide Oligo Design}

Three DNA guide oligos, which will serve as the template for gRNAs, were designed for each tet gene using CRISPRscan (https://www.crisprscan.org/) (Table 1) (see Note 1) $[21,22]$ (Fig. 1a). The DNA guide oligos were selected based on their protospacer sequence score, appearance in the first half of the gene, appearance in all annotated isoforms, and lack of published single nucleotide polymorphisms (SNPs) (Table 2). The protospacer sequences for each gene were also kept within a $300 \mathrm{bp}$ window, to allow for easier and more accurate knockout calculations, as they can be covered by a single PCR amplicon. Each DNA guide oligo (Table 1) starts with a T7 RNA polymerase transcription start site $\left(5^{\prime}\right.$-taatacgactcactata- $\left.3^{\prime}\right)$, followed by the protospacer sequence with the first two nucleotides changed to guanine for efficient transcription (Table 2), and ends with a sequence complementary to the universal DNA oligo $\left(5^{\prime}\right.$-gttttagagctagaa- $\left.3^{\prime}\right)$. The universal DNA oligo contains the structural template for each gRNA and will be annealed to DNA guide oligos in Subheading 3.2.

In order to create templates for gRNA in vitro transcription in Subheading 3.3, the DNA guide oligos and the universal oligo need to be annealed and amplified.

1. Resuspend the DNA guide oligos (Table 1) and the universal DNA oligo at a concentration of $100 \mu \mathrm{M}$ in nuclease-free water. Mix well by vortexing. From $100 \mu \mathrm{M}$ stock solutions prepare $10 \mu \mathrm{M}$ working solutions using nuclease-free water. Mix well by vortexing.

2. In separate $0.2 \mathrm{ml}$ PCR tubes, add $2 \mu \mathrm{l}$ of each guide oligo $(10 \mu \mathrm{M})$. There should be nine tubes in total.

3 . Create a master mix by combining $2 \mu \mathrm{l}$ of universal DNA oligo $(10 \mu \mathrm{M}), 5 \mu \mathrm{l} 10 \times$ PCR buffer, $2 \mu \mathrm{l} \mathrm{MgCl}_{2}(50 \mathrm{mM}), 1 \mu \mathrm{l} \mathrm{dNTP}$ $(10 \mathrm{mM}), 0.2 \mu \mathrm{l}$ Taq DNA Polymerase $(5 \mathrm{U} / \mu \mathrm{l})$, and $37.8 \mu \mathrm{l}$ nuclease-free water per reaction. Mix gently by pipetting.

4. Add $48 \mu \mathrm{l}$ of the master mix to each $0.2 \mathrm{ml}$ PCR tube. Mix gently by pipetting. 


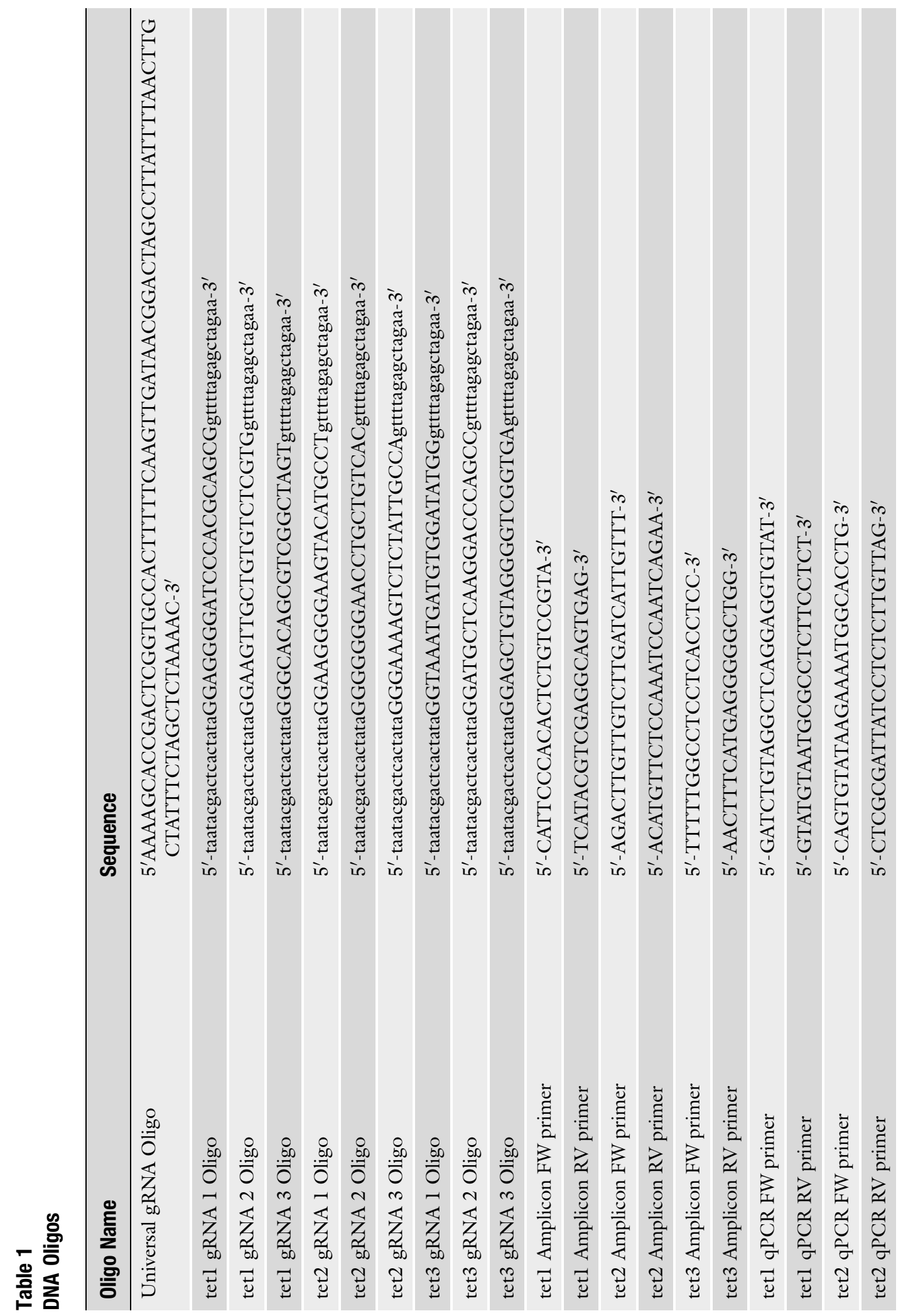




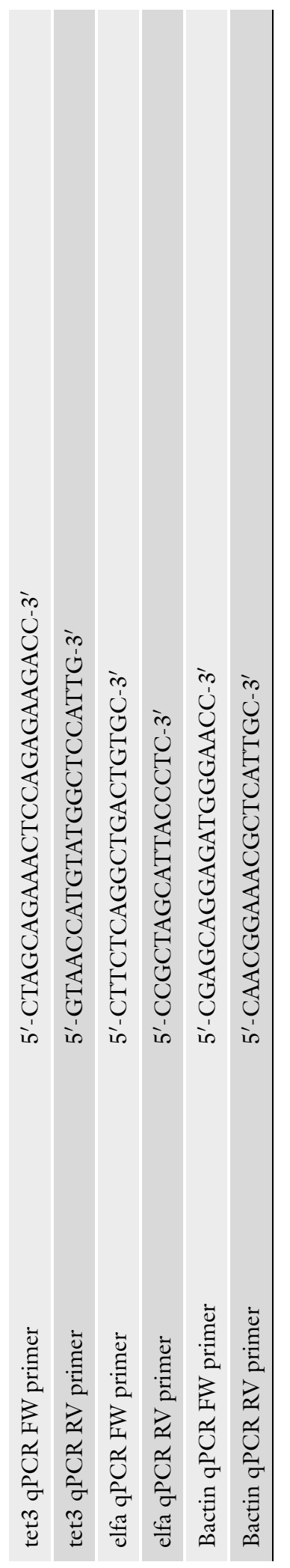




\section{Table 2}

gRNA oligo design for tet1/2/3 KO zebrafish

\begin{tabular}{|lllc}
\hline Oligo Name & oligo Score & Protospacer & Exon \\
\hline tet1 gRNA 1 Oligo & 57 & GGAGGGGATCCCACGCAGCG & 5 \\
\hline tet1 gRNA 2 Oligo & 81 & AGAAGTTGCTGTGTCTCGTG & 5 \\
\hline tet1 gRNA 3 Oligo & 65 & GGGCACAGCGTCGGCTAGT & 5 \\
\hline tet2 gRNA 1 Oligo & 64 & AGAAGGGGAAGTACATGCCT & 1 \\
\hline tet2 gRNA 2 Oligo & 81 & GAGGGGGAACCTGCTGTCAC & 1 \\
\hline tet2 gRNA 3 Oligo & 24 & AGGAAAAGTCTCTATTGCCATGG & 1 \\
\hline tet3 gRNA 1 Oligo & 54 & AGTAAATGATGTGGATATGG & 4 \\
\hline tet3 gRNA 2 Oligo & 43 & GTATGCTCAAGGACCCAGCC & 4 \\
\hline tet3 gRNA 3 Oligo & 68 & TGAGCTGTAGGGGTCGGTGA & 4 \\
\hline
\end{tabular}

5. Anneal and amplify the samples in a thermocycler under the following conditions:

(a) 3 min at $95^{\circ} \mathrm{C}$

(b) 30 cycles of $30 \mathrm{~s}$ at $95^{\circ} \mathrm{C}, 30 \mathrm{~s}$ at $45^{\circ} \mathrm{C}, 20 \mathrm{~s}$ at $72{ }^{\circ} \mathrm{C}$

(c) $5 \mathrm{~min}$ at $72{ }^{\circ} \mathrm{C}$

(d) Hold at $4{ }^{\circ} \mathrm{C}$

6. In the last $30 \mathrm{~min}$ of the PCR program, remove an aliquot of AMPure XP beads from the fridge and let it equilibrate at room temperature ( see Note 2). Also prepare fresh 75\% ethanol.

7. After the program has finished, add $90 \mu \mathrm{l}$ of room temperature AMPure XP beads (1.8:1 ratio of beads to sample) to each of the $0.2 \mathrm{ml}$ PCR tubes. Mix well by pipetting until the solution looks homogenous.

8. Incubate the PCR tubes at room temperature for $10 \mathrm{~min}$.

9. Place the PCR tubes on a magnetic stand and leave at room temperature for 5 min or until the liquid appears clear.

10. Remove and discard the supernatant from the PCR tubes (see Note 3).

11. Carefully add $200 \mu$ of $75 \%$ ethanol to each PCR tube and then transfer the tubes back and forth between neighboring positions in the magnetic stand, allowing the beads to travel through the ethanol.

12. Remove and discard all of the supernatant from the PCR tubes.

13. Repeat steps 11 and $\mathbf{1 2}$.

14. Allow beads to air-dry until no ethanol is visible. 
15. Elute the DNA by adding $21 \mu$ l nuclease-free water to each sample. Mix well by pipetting until the beads are completely resuspended (see Note $\mathbf{4}$ ).

16. Place the PCR tubes on a magnetic rack for $5 \mathrm{~min}$ at room temperature.

17. Transfer $20 \mu \mathrm{l}$ of the supernatant from each sample to new $0.2 \mathrm{ml}$ PCR tubes being careful not to carry over any beads.

\section{3 gRNA In Vitro Transcription}

1. In separate $0.2 \mathrm{ml}$ PCR tubes, add $6.3 \mu \mathrm{l}$ of each annealed template oligo from Subheading 3.2.

2. Using the AMPLISCRIBEтм T7-FLASH ${ }^{\text {тм }}$ Transcription Kit, create a master mix by combining $2 \mu \mathrm{l} 10 \times$ reaction buffer, $1.8 \mu \mathrm{l}$ ATP $(100 \mathrm{mM}), 1.8 \mu \mathrm{l}$ GTP $(100 \mathrm{mM}), 1.8 \mu \mathrm{l}$ CTP

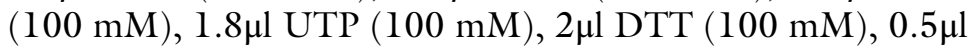
RNase inhibitor, and $2 \mu \mathrm{l} 7 \mathrm{RNA}$ polymerase per reaction. It is important that the reagents are added in that exact order to avoid precipitation of the components. Mix well by pipetting.

3. Add $13.7 \mu$ of the master mix to each PCR tube. Mix well by pipetting.

4. Incubate the PCR tubes at $42{ }^{\circ} \mathrm{C}$ overnight in a thermocycler with lid heating $\mathrm{ON}$.

5. After the overnight incubation, add 1.5 $\mu$ l DNaseI to each PCR tube and incubate at $37^{\circ} \mathrm{C}$ for $25 \mathrm{~min}$.

6. Add $80 \mu \mathrm{l}$ nuclease-free water to each PCR tube. Mix well by pipetting.

7. Transfer the total $100 \mu \mathrm{l}$ of each sample to separate $1.5 \mathrm{ml}$ nuclease-free tubes ( see Note $\mathbf{5}$ ).

8. Precipitate the gRNA by adding $10 \mu$ of Sodium Acetate $(3 \mathrm{M}$, $\mathrm{pH} 5.5)$ and $300 \mu \mathrm{l}$ of $100 \%$ ethanol to each $1.5 \mathrm{ml}$ tube. Incubate overnight at $-20{ }^{\circ} \mathrm{C}$ or $1-2 \mathrm{~h}$ at $-80{ }^{\circ} \mathrm{C}$.

9. After the incubation, pellet the gRNAs by centrifugation at max speed $(>12,000 \times g)$ for $30 \mathrm{~min}$ at $4{ }^{\circ} \mathrm{C}$.

10. Wash the gRNA pellets by adding $500 \mu$ of $75 \%$ ethanol (see Note 6).

11. Repeat step $\mathbf{1 0}$ for a total of two washes.

12. Air-dry the gRNA pellets until no ethanol is visible.

13. Resuspend the gRNA pellets in $50 \mu \mathrm{l}$ nuclease-free water, pipetting up and down at least 20 times. Incubate the tubes on ice for $15 \mathrm{~min}$, gently flicking the tube every $5 \mathrm{~min}$ to further promote resuspension. 


\subsection{Microinjection Chambers and Needles}

14. Measure the RNA concentration using Qubit ${ }^{\mathrm{TM}}$ RNA BR Assay Kit. Expected concentrations range from $500 \mathrm{ng} / \mu \mathrm{l}$ to $3 \mu \mathrm{g} / \mu \mathrm{l}$.

15. Make $5 \mu \mathrm{l}$ aliquots out of each tet gRNA and store them at $-80^{\circ} \mathrm{C}$. The gRNAs are now ready for microinjections.

In order to perform microinjections, both microinjection needles and microinjection chambers need to be made. It is recommended to perform this step on the day before microinjections.

1. Prepare $100 \mathrm{ml}$ of hot $2 \%$ agarose by dissolving $2 \mathrm{~g}$ of agarose in $100 \mathrm{ml}$ of water. Microwave the mixture appropriately until the agarose is dissolved.

2. Pour approximately $20 \mathrm{ml}$ of the hot $2 \%$ agarose into two petri dishes $(90 \mathrm{~mm} \times 15 \mathrm{~mm})$ and let them cool on the bench at room temperature.

3 . Once the agar has nearly solidified, add $20 \mathrm{ml}$ of the $2 \%$ agarose to each dish.

4. Immediately place the larval injection mold (zebrafish microinjection and transplantation mold kit) teeth into the liquid (see Note 7).

5. After the agarose hardens, remove the plastic mold and wrap the Petri dish in parafilm. This can be stored for up to 7 days at $4{ }^{\circ} \mathrm{C}$.

6. Prepare needles for microinjection by loading a glass capillary $(0.78 \mathrm{~mm}$, od: $1 \mathrm{~mm})$ into the Sutter Instrument. Pull the glass capillary into two equal sized needles using the following machine setting: Heat: 480, Pull: 200, Vel: 100, Time: 225 .

7. Repeat step 6 two times until there are six pulled needles.

8. Under a microscope, cut approximately $1 \mathrm{~mm}$ off the sharp end of each microinjection needle using a scalpel blade. Store the needles in a large petri dish $(150 \mathrm{~mm} \times 15 \mathrm{~mm})$ on top of a bed of Blu Tack adhesive or tape.

3.5 Zebrafish Egg Collection and Preparation of the tet1/2/3 KO Microinjection Mixture
In this step, zebrafish are set up for mating and the tetl/2/3 KO microinjection mixture is prepared. The single microinjection mixture will contain all $9 \mathrm{gRNAs}$ created in Subheading 3.3.

1. The evening before the microinjections, set up two zebrafish mating tanks. Each mating tank is to contain 4 males and 2 females separated by a divider. More tanks can be set up if desired (see Note 8).

2. On the morning of microinjections, prepare tet $1 / 2 / 3 \mathrm{KO}$ microinjection mixture by combining $100 \mathrm{ng}$ of each tet gRNA with $1 \mu \mathrm{l}$ Cas 9 nuclease (NEB) and $1.5 \mu \mathrm{KCl}(1 \mathrm{M})$ in a single nuclease-free tube. Top the up mixture to $5 \mu \mathrm{l}$ with nuclease-free water and mix by pipetting. 
3. Incubate the microinjection mixture at $37{ }^{\circ} \mathrm{C}$ for $5 \mathrm{~min}$ to allow the complex between Cas9 nuclease and the gRNAs to form.

4. During incubation, take the microinjection chambers out of the fridge to warm up.

5. After incubation, add $1 \mu \mathrm{l}$ of $0.5 \%$ phenol red to the injection mixture. Mix by pipetting.

6. After the microinjection mixture is prepared, transfer the zebrafish from one of the mating tanks from step $\mathbf{l}$ to a new mating tank and remove the dividers.

7. Observe the zebrafish until the females begin to release eggs. After the first eggs have been released, wait $10 \mathrm{~min}$ to allow for fertilization to occur.

8. Collect all eggs using a mesh strainer. The zebrafish can then be moved to a new tank and separated again by a divider.

9. Clean the eggs with E3 solution before transferring them to a petri dish $(90 \mathrm{~mm} \times 15 \mathrm{~mm})$. If there appears to be at least $\sim 400$ produced eggs, proceed to microinjections. If not, repeat steps 5-9 with the other mating tank (see Note 9).

\subsection{Microinjections}

1. Turn on gas supply for the Picospritzer III.

2. Turn on the Picospritzer III and set the pressure to 20 PSI and the pulse duration to $20 \mathrm{~ms}$.

3. Using a microloader pipette tip, load one of the cut microinjection needles (Subheading 3.4) with $2 \mu$ l of the microinjection mixture (Subheading 3.5).

4. Attach the loaded microinjection needle to the needle holder on the Picospritzer III.

5. Add a droplet of mineral oil to the center of a stage microruler and place it under a microscope at approximately $6 \times$ magnification.

6. Place the tip of a loaded microinjection needle into the mineral oil and dispense the microinjection mixture by pressing down on the foot pedal. Under the microscope, measure the size of the red bead which forms in the mineral oil on the stage microruler.

7. Adjust the pulse duration on the picospritzer III until a bead with a diameter of $0.1 \mathrm{~mm}$ can be produced. A bead with a diameter of $0.1 \mathrm{~mm}$ equates to $500 \mathrm{pL}$ of microinjection mixture. Further cutting of the needle will be required if a maximum of $60 \mathrm{~ms}$ pulse duration is not enough.

8. Using a plastic pipette, transfer $\sim 200$ zebrafish embryos into the wells of the microinjection chamber. 


\subsection{Embryo Collection}

\section{8 gDNA and RNA Extraction}

9. Observe the eggs under a microscope and confirm that the eggs are not past the first cell stage.

10. Inject a single egg by smoothly piercing the chorion with the microinjection needle until the tip enters into the cell or yolk ( see Note 10). Press down on the foot pedal while the needle is inside the egg. The red injection mixture should be visible inside the egg if the injection was successful (see Note 11).

11. Repeat the injections for every egg in the chamber.

12. Using E3 solution, rinse the eggs off into a new petri dish and incubate them in an incubator at $29^{\circ} \mathrm{C}$.

13. Transfer the remaining $\sim 200$ uninjected eggs to the same incubator to use as uninjected controls.

14. In the evening, clean the embryos by removing all of the dead and unfertilized eggs, and by replacing the E3 solution with a plastic pipette.

1. The next morning, $24 \mathrm{~h}$ after the eggs were laid, observe both injected and uninjected embryos under a microscope. tet $1 / 2 / 3$ KO embryos should display phenotypes such as microphthalmia and should lack properly formed midbrain and hindbrain boundaries (Fig. 1b) (see Note 12).

2. Collect four batches of 20 embryos that display developmental phenotypes from the injected eggs and four batches of 20 embryos from the uninjected control in eight separate $1.5 \mathrm{ml}$ nuclease-free tubes.

3. Remove all the liquid from the $1.5 \mathrm{ml}$ tubes and either snapfreeze the embryos in liquid nitrogen or immediately proceed to DNA and RNA extraction (see Note 13).

In this step, gDNA is extracted from two batches of 20 embryos from both the injected and uninjected samples. These samples will be used as biological replicates in amplicon sequencing and RRBS and are referred to now on as tet $1 / 2 / 3 \mathrm{KO}$ replicate 1 , tet $1 / 2 / 3 \mathrm{KO}$ replicate 2 , uninjected replicate 1 , and uninjected replicate 2 . RNA is also extracted from the remaining embryo batches for use in qPCR estimation of knockout efficiencies.

1. Extract gDNA from two batches of 20 embryos for both injected and uninjected samples using the Qiagen blood and Tissue DNA extraction kit.

2. Elute the gDNA in $50 \mu \mathrm{l}$ nuclease-free water and measure the concentration using Qubit ${ }^{\mathrm{TM}}$ DNA HS Assay Kit. Store the samples at $-20^{\circ} \mathrm{C}$ for later use in RRBS and amplicon sequencing in Subheadings 3.9 and 3.10. 
A
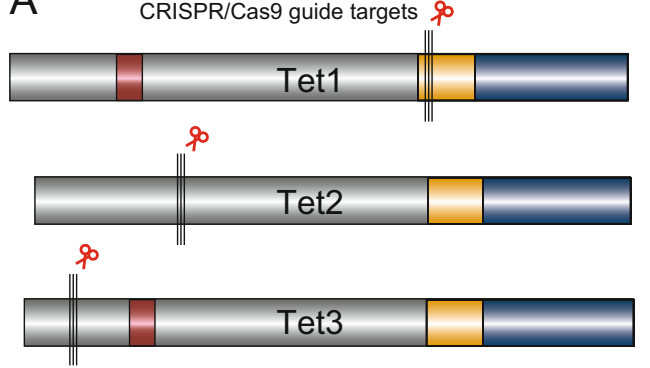

CXXC domain cysteine-rich

DSBH (dioxygenase activity / Fe(II) binding)
C

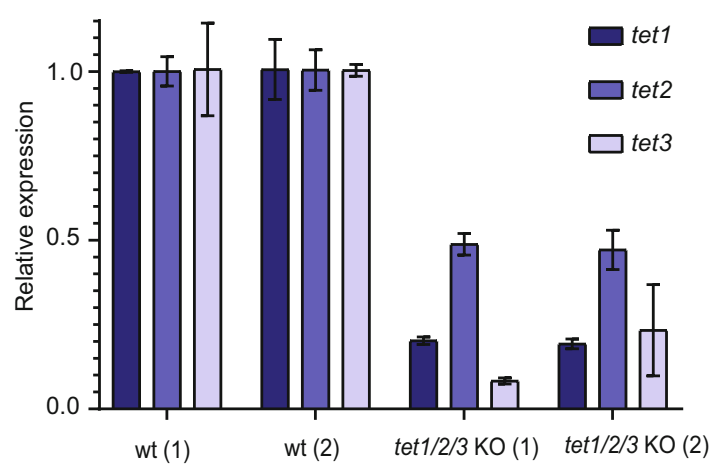

B

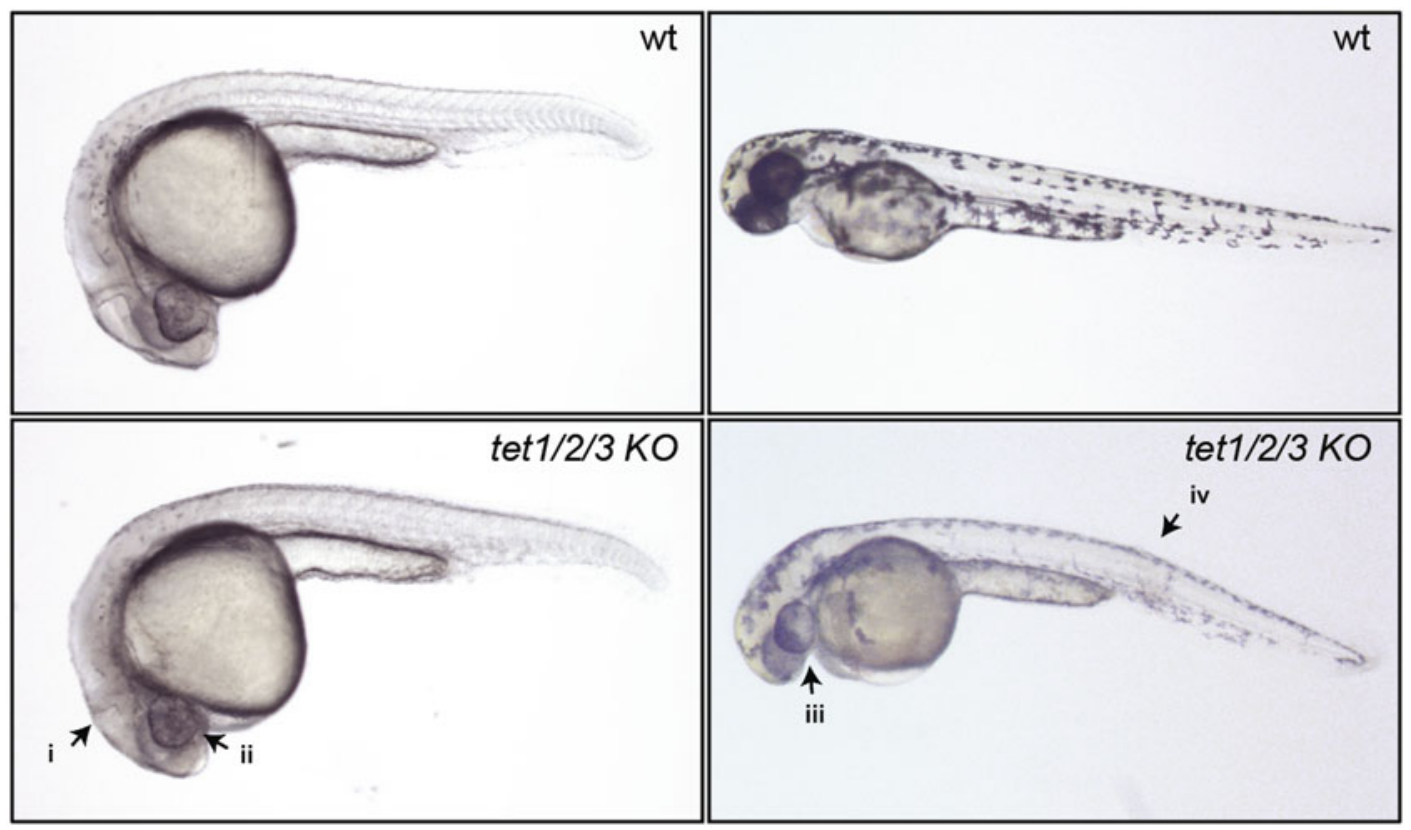

Fig. 1 Phenotypes of tet1/2/3KO F0 zebrafish following CRISPR/Cas9 microinjections. (a) Zebrafish Tet protein structure and guide RNA design. (b) Top left: uninjected $24 \mathrm{hpf}$ zebrafish, bottom left: tet1/2/3 KO injected $24 \mathrm{hpf}$ zebrafish displaying improperly formed midbrain hindbrain boundary (i) and microphthalmia (ii). Top right: uninjected $48 \mathrm{hpf}$ zebrafish; bottom right: tet1/2/3 K0 injected $48 \mathrm{hpf}$ zebrafish displaying microphthalmia (iii), altered pigmentation and curved trunk (iv). (c) gPCR quantification of two biological replicates of uninjected wild type (wt) and injected (tet1/2/3 K0) F0 zebrafish. Each gene is tested in triplicate and represented as the mean relative expression to wt. Error bars represent standard deviation

3. Under a fume hood, add $250 \mu$ lof TRIsure reagent to the two remaining $1.5 \mathrm{ml}$ tubes for both the uninjected and injected groups.

4. Homogenize the embryos using a pestle or needle. 
5. Once sufficiently homogenized, add a further $750 \mu$ l TRIsure reagent to each tube and incubate for $5 \mathrm{~min}$ at room temperature.

6. After the incubation, add $0.2 \mathrm{ml}$ chloroform to each tube and mix by inverting the tube several times. Incubate the sample for $5 \mathrm{~min}$ at room temperature.

7. Centrifuge the samples at $12,000 \times g$ for $15 \mathrm{~min}$ at $4^{\circ} \mathrm{C}$.

8. The mixture will separate into a lower organic phase, an interphase, and a colorless upper aqueous phase. Transfer the top aqueous layers into new $1.5 \mathrm{ml}$ nuclease-free tubes. It is better to be conservative and take approximately $75 \%$ of the aqueous layer then risk contamination with other layers.

9. Add $0.5 \mathrm{ml}$ isopropanol to each $1.5 \mathrm{ml}$ tube in order to precipitate the RNA. Incubate at room temperature for $10 \mathrm{~min}$.

10. Pellet the RNA by centrifugation at $12,000 \times g$ for $10 \mathrm{~min}$ at $4{ }^{\circ} \mathrm{C}$.

11. Remove the supernatant with a pipette and add $1 \mathrm{ml} 75 \%$ ethanol.

12. Mix the sample by inversion and re-pellet the RNA by centrifugation at $12,000 \times g$ for $5 \mathrm{~min}$ at $4{ }^{\circ} \mathrm{C}$.

13. Remove as much ethanol as possible and allow the samples to air-dry at room temperature until no liquid is visible.

14. Resuspend the pellet by adding $20 \mu \mathrm{l}$ of nuclease-free water, pipetting up and down at least 20 times gently. Incubate the RNA on ice for $15 \mathrm{~min}$, gently flicking the tube every $5 \mathrm{~min}$ to further facilitate resuspension.

15. Measure the concentration of RNA using the Qubit ${ }^{\mathrm{TM}}$ RNA BR Assay Kit as well as a Nanodrop to estimate the quality of the sample. A 260/280 ratio of $\sim 2$ and a 260/230 ratio $>2$ is desired for efficient cDNA synthesis and qPCR.

3.9 Amplicon Sequencing
1. Using $1 \mu \mathrm{l}$ of the tet $1 / 2 / 3 \mathrm{KO}$ gDNA from Subheading 3.8, set up six $0.2 \mathrm{ml}$ PCR tubes as outlined in Table 3.

2. Make a PCR master mix by combining $5 \mu \mathrm{l} 10 \times$ PCR Buffer, $2 \mu \mathrm{lgCl}_{2}(10 \mathrm{mM}), 1 \mu \mathrm{l} \mathrm{dNTP}(10 \mathrm{mM}), 0.2 \mu \mathrm{l}$ Taq DNA polymerase $(5 \mathrm{U} / \mu \mathrm{l})$, and $39.8 \mu \mathrm{l}$ nuclease-free water in $1.5 \mathrm{ml}$ microfuge tube per reaction. Mix by pipetting.

3. Add $48 \mu \mathrm{l}$ of master mix to each PCR tube and mix gently by pipetting.

4. Amplify each amplicon under the following conditions in a thermocycler:

(a) 3 min at $95^{\circ} \mathrm{C}$.

(b) 30 cycles of $30 \mathrm{~s}$ at $95^{\circ} \mathrm{C}, 30 \mathrm{~s}$ at $45^{\circ} \mathrm{C}, 20 \mathrm{~s}$ at $72{ }^{\circ} \mathrm{C}$. 


\section{Table 3}

Amplicon sequencing setup

\begin{tabular}{|c|c|c|c|}
\hline & gDNA & Forward Primer $(10 \mu \mathrm{M})$ & Reverse Primer(10 $\mu \mathrm{M})$ \\
\hline 1 & $1 \mu \mathrm{l}$ tet $1 / 2 / 3 \mathrm{KO}$ replicate 1 & $0.5 \mu \mathrm{l}$ tet 1 Amplicon $\mathrm{F}$ & $0.5 \mu \mathrm{l}$ tet 1 Amplicon $\mathrm{R}$ \\
\hline 2 & $1 \mu \mathrm{l}$ tet $1 / 2 / 3 \mathrm{KO}$ replicate 1 & $0.5 \mu \mathrm{l}$ tet 2 Amplicon $\mathrm{F}$ & $0.5 \mu \mathrm{l}$ tet 2 Amplicon $\mathrm{R}$ \\
\hline 3 & $1 \mu \mathrm{l}$ tet $1 / 2 / 3 \mathrm{KO}$ replicate 1 & $0.5 \mu \mathrm{l}$ tet3 Amplicon $\mathrm{F}$ & $0.5 \mu \mathrm{l}$ tet3 Amplicon $\mathrm{R}$ \\
\hline 4 & $1 \mu \mathrm{ltet} 1 / 2 / 3 \mathrm{KO}$ replicate 2 & $0.5 \mu \mathrm{l}$ tet 1 Amplicon $\mathrm{F}$ & $0.5 \mu \mathrm{l}$ tet 1 Amplicon $\mathrm{R}$ \\
\hline 5 & $1 \mu \mathrm{l}$ tet $1 / 2 / 3 \mathrm{KO}$ replicate 2 & $0.5 \mu \mathrm{l}$ tet 2 Amplicon $\mathrm{F}$ & $0.5 \mu \mathrm{l}$ tet 2 Amplicon $\mathrm{R}$ \\
\hline 6 & $1 \mu \mathrm{l}$ tet $1 / 2 / 3 \mathrm{KO}$ replicate 2 & $0.5 \mu \mathrm{l}$ tet3 Amplicon $\mathrm{F}$ & $0.5 \mu \mathrm{l}$ tet3 Amplicon $\mathrm{R}$ \\
\hline
\end{tabular}

(c) 5 min at $72^{\circ} \mathrm{C}$.

(d) Hold at $4{ }^{\circ} \mathrm{C}$.

5. In the last $30 \mathrm{~min}$ of the PCR program, remove an aliquot of AMPure XP beads from the fridge and let it equilibrate at room temperature. Also prepare fresh $75 \%$ ethanol.

6. Add $80 \mu \mathrm{l}$ of room temperature AMPure XP beads to the $50 \mu \mathrm{l}$ annealed products and gently mix by pipetting.

7. Incubate the PCR tubes at room temperature for $10 \mathrm{~min}$.

8. Place the PCR tubes on a magnetic stand and keep at room temperature for 5 min or until the liquid appears clear.

9. Remove and discard the supernatant from each PCR tube.

10. Carefully add $200 \mu \mathrm{l}$ of $75 \%$ ethanol to each PCR tube and then transfer the tubes back and forth between neighboring positions in the magnetic stand, allowing the beads to travel through the ethanol.

11. Remove and discard all of the supernatant from the PCR tubes.

12. Repeat steps $\mathbf{1 0}$ and $\mathbf{1 1 .}$

13. Allow beads to air-dry until no ethanol is visible.

14. Elute the DNA by adding $17 \mu 1$ nuclease-free water to each tube. Mix well by pipetting until the beads are completely resuspended.

15. Place the PCR tubes on magnetic rack for $5 \mathrm{~min}$ at room temperature.

16. Transfer $16 \mu \mathrm{l}$ of the supernatant from each sample to new $0.2 \mathrm{ml}$ PCR tubes.

17. Add $1 \mu \mathrm{l}$ NEXTflex barcode adapter $26(12.5 \mu \mathrm{M})$ to the first three tubes containing replicate 1 amplicons, and $1 \mu$ NEXTflex barcode adapter $44(12.5 \mu \mathrm{M})$ to the remaining three tubes containing replicate 2 amplicons (see Note 14). 


\subsection{RRBS: Mspl Digestion}

3.11 RRBS: End Repair and A-Tailing
18. Add $2 \mu \mathrm{l}$ T4 DNA Ligase Buffer $\times 10$ and $1 \mu \mathrm{l} 44$ DNA Ligase $400 \mathrm{U} / \mu \mathrm{l}$ to each tube. Mix by pipetting.

19. Incubate the ligation mixtures at $16{ }^{\circ} \mathrm{C}$ overnight in a thermocycler.

20. Heat-inactivate the samples at $65^{\circ} \mathrm{C}$ for $10 \mathrm{~min}$.

21. Transfer $1 \mu$ from each ligation mixture to new $0.2 \mathrm{ml} \mathrm{PCR}$ tubes.

22. Create a PCR master mix by combining $1 \mu l$ of NEXTflex universal primer $(10 \mu \mathrm{M}), 5 \mu \mathrm{l} 10 \times$ PCRBuffer, $2 \mu \mathrm{l} \mathrm{MgCl}_{2}$ $(10 \mathrm{mM}), 1 \mu \mathrm{l}$ DNTP $(10 \mathrm{mM}), 0.2 \mu \mathrm{l}$ Taq DNA polymerase $(5 \mathrm{U} / \mu \mathrm{l})$, and $39.8 \mu \mathrm{l}$ nuclease-free water in a $1.5 \mathrm{ml}$ nucleasefree tube per reaction. Mix by pipetting.

23. Add $49 \mu \mathrm{l}$ of the PCR master mix to the $0.2 \mathrm{ml}$ PCR tubes. Mix by pipetting.

24. Amplify amplicons with successfully ligated adapters by using the following conditions in a thermocycler:
(a) 3 min at $95^{\circ} \mathrm{C}$.
(b) 30 cycles of $30 \mathrm{~s}$ at $95^{\circ} \mathrm{C}, 30 \mathrm{~s}$ at $45^{\circ} \mathrm{C}, 20 \mathrm{~s}$ at $72^{\circ} \mathrm{C}$.
(c) 5 min at $72{ }^{\circ} \mathrm{C}$.
(d) Hold at $4{ }^{\circ} \mathrm{C}$

25. Purify the DNA by repeating steps 6-15.

26. Measure the DNA concentration using the Qubit ${ }^{\mathrm{TM}}$ DNA HS Assay Kit. Store the samples at $-20^{\circ} \mathrm{C}$.

RRBS libraries will be made from all four gDNA samples from Subheading 3.8: tetl $/ 2 / 3 \mathrm{KO}$ replicate 1 , tet $1 / 2 / 3 \mathrm{KO}$ replicate 2 , uninjected replicate 1 , and uninjected replicate 2 .

1. Create a working solution of $5 \mathrm{ng} / \mu \mathrm{l}$ unmethylated lambda DNA.

2. Digest $500 \mathrm{ng}$ of all four gDNA samples from Subheading 3.9 by combining them with $5 \mu \mathrm{l}$ CutSmart buffer, $1 \mu \mathrm{l}$ Mspl $(20 \mathrm{U} / \mu \mathrm{l})$, and $0.5 \mu \mathrm{l} 5 \mathrm{ng} / \mu \mathrm{l}$ unmethylated lambda DNA in separate $0.2 \mathrm{ml}$ PCR tubes. Top each reaction up to $50 \mu \mathrm{l}$ with nuclease-free water ( see Note 15).

3. Digest the reactions at $37^{\circ} \mathrm{C}$ overnight in a thermocycler with lid heating set to $50^{\circ} \mathrm{C}$.

4. Heat-inactive the reactions at $80{ }^{\circ} \mathrm{C}$ for $20 \mathrm{~min}$ then hold at $4{ }^{\circ} \mathrm{C}$.

MspI digestion creates fragments with identical 2 bp $5^{\prime}$ overhangs. As a result, only a Klenow fragment is needed for both end repairing and A-tailing in RRBS libraries. 
1. To each $50 \mu \mathrm{l}$ of digested gDNA from Subheading 3.10, add $1.5 \mu \mathrm{l}$ dNTP $(1 \mathrm{mM}), 1.5 \mu \mathrm{l}$ dATP $(10 \mathrm{mM}), 1.5 \mu \mathrm{l}$ Klenow Fragment $\left(3^{\prime} \rightarrow 5^{\prime}\right.$ exo $\left.{ }^{-}, 5 \mathrm{U} / \mu \mathrm{l}\right), 0.5 \mu \mathrm{l}$ CutSmart buffer. Mix by pipetting.

2. Fill in the overhangs and A-tail the fragments by incubating the reactions at $37^{\circ} \mathrm{C}$ for $50 \mathrm{~min}$ in a thermocycler.

3. Heat-inactivate the reactions at $80^{\circ} \mathrm{C}$ for $30 \mathrm{~min}$ then hold at $4{ }^{\circ} \mathrm{C}$.

3. At the beginning of the heat-inactivation step, remove an aliquot of AMPure XP beads from the fridge and let it equilibrate at room temperature. Also prepare fresh $75 \%$ ethanol.

4. Add $71.5 \mu$ l of room temperature AMPure XP beads to the $55 \mu \mathrm{l}$ annealed products (1.3:1 ratio of beads to sample) and gently mix by pipetting until the solution is homogenous.

5. Incubate the PCR tubes at room temperature for $10 \mathrm{~min}$.

6. Place the PCR tubes on a magnetic stand and keep at room temperature for $\mathbf{5}$ min or until the liquid appears clear.

7. Remove and discard the supernatant from each PCR tube.

8. Carefully add $200 \mu \mathrm{l}$ of $75 \%$ ethanol to each tube and then transfer the tubes back and forth between neighboring positions in the magnetic stand, allowing the beads to travel through the ethanol.

9. Remove and discard all of the supernatant from the PCR tubes.

10. Repeat steps 32 and 33.

11. Allow beads to air-dry until no ethanol is visible.

12. Elute the DNA by adding $17 \mu$ l nuclease-free water to each sample. Mix well by pipetting until the beads are completely resuspended.

13. Place the PCR tubes on a magnetic rack and let stand for $5 \mathrm{~min}$ at room temperature.

14. Transfer $16 \mu \mathrm{l}$ of the supernatant from each sample to new $0.2 \mathrm{ml}$ PCR tubes.

3.12 RRBS: Adaptor Ligation
1. To the eluted DNA from Subheading 3.11, add $2 \mu 110 \times \mathrm{T} 4$ DNA Ligase Buffer, $1 \mu \mathrm{l}$ T4 DNA Ligase $400 \mathrm{U} / \mu \mathrm{l}$, and $1 \mu \mathrm{l}$ NEXTflex barcode adapter $(12.5 \mu \mathrm{M})$ as outlined in Table 4 (see Note 16).

2. Ligate the adapters to the DNA fragments by incubating the reactions at $16{ }^{\circ} \mathrm{C}$ overnight followed by heat inactivation at $65{ }^{\circ} \mathrm{C}$ for $10 \mathrm{~min}$, in a thermocycler.

3. Add $30 \mu \mathrm{l}$ of nuclease-free water to each sample bringing them up to a total volume of $50 \mu \mathrm{l}$ each. 
Table 4

RRBS Barcode Adapters

\begin{tabular}{ll}
\hline Sample & NEXTflex barcode adapter \\
\hline Uninjected replicate 1 & $\mathbf{2 4}$ \\
\hline Uninjected replicate 2 & $\mathbf{3 1}$ \\
\hline tet $1 / 2 / 3$ KO replicate 1 & $\mathbf{3 8}$ \\
tet $1 / 2 / 3$ KO replicate 2 & $\mathbf{4 1}$ \\
\hline
\end{tabular}

4. Add $65 \mu \mathrm{l}$ of room temperature AMPure XP beads to the $50 \mu \mathrm{l}$ annealed products (1.3:1 ratio of beads to sample) and gently mix by pipetting until the solution is homogenous.

5. Incubate the PCR tubes at room temperature for $10 \mathrm{~min}$.

6. Place the PCR tubes on a magnetic stand and keep at room temperature for $\mathbf{5}$ min or until the liquid appears clear.

7. Remove and discard the supernatant from each PCR tube.

8. Carefully add $200 \mu$ l of $75 \%$ ethanol to each tube and then transfer the tubes back and forth between neighboring positions in the magnetic stand, allowing the beads to travel through the ethanol.

9. Remove and discard all of the supernatant from the PCR tubes.

10. Repeat steps 8 and $\mathbf{9 .}$

11. Allow beads to air-dry until no ethanol is visible.

12. Elute the DNA by adding $21 \mu$ l nuclease-free water to each sample. Mix well by pipetting until the beads are completely resuspended.

13. Place the PCR tubes on magnetic rack and let stand for 5 min at room temperature.

14. Transfer $20 \mu \mathrm{l}$ of the supernatant from each sample to new $0.2 \mathrm{ml}$ PCR tubes.

3.13 RRBS: Bisulfite Conversion
1. Make the CT conversion reagent according to manufacturer's instructions in the EZ DNA Methylation-Gold kit.

2. Add $130 \mu \mathrm{l}$ of CT conversion reagent to each sample from Subheading 3.12. Mix gently by pipetting.

3. Split the $150 \mu$ l of each sample from step 2 into $3 \times 50 \mu l$ in $0.2 \mathrm{ml}$ PCR tubes. This assures the volume will be completely covered by the wells of the thermocycler.

4. Perform the bisulfite conversion, column purification, and desulfonation according to the manufacturer's instructions in the EZ DNA Methylation-Gold kit.

5 . Elute each sample in $24 \mu \mathrm{l}$ nuclease-free water. 
3.14 RRBS: PCR Amplification

\subsection{RRBS: Library Quantification and Multiplexing}

1. Transfer $24 \mu \mathrm{l}$ of the bisulfite-converted samples from Subheading 3.13 to separate $0.2 \mathrm{ml}$ PCR tubes.

2. Add $1 \mu \mathrm{l}$ of $12.5 \mu \mathrm{M}$ NEXTflex universal primer and $25 \mu \mathrm{l}$ $2 \times$ KAPA HiFi HotStart Uracil + ReadyMix to each PCR tube. Mix gently by pipetting.

3. Perform a low amplification PCR under the following conditions in a thermocycler:
(a) 2 min at $95^{\circ} \mathrm{C}$.
(b) 13 cycles of $20 \mathrm{~s}$ at $98^{\circ} \mathrm{C}, 15 \mathrm{~s}$ at $60^{\circ} \mathrm{C}, 1$ min at $72{ }^{\circ} \mathrm{C}$.
(c) 5 min at $72{ }^{\circ} \mathrm{C}$.
(d) Hold reaction at $4{ }^{\circ} \mathrm{C}$.

4. Purify the samples with AMPure XP beads as in Subheading 3.12 .

5. Measure the DNA concentration of each RRBS library using the Qubit ${ }^{\mathrm{TM}}$ DNA HS Assay Kit. The expected concentration will be approximately between 10 and $30 \mathrm{ng} / \mu \mathrm{l}$.

6. Run $1 \mu l$ of each library on a Tapestation D1000 ScreenTape following manufacturer's instructions to assess the fragment size distribution of each library (see Note 17).

In the next step, the amplifiable DNA in each library will be quantified by qPCR against DNA standards. This will allow the pooling of the samples in appropriate ratios to ensure even sequencing depth for each library.

1. Take $1 \mu \mathrm{l}$ of each RRBS library from Subheading 3.15 and dilute it by adding $99 \mu \mathrm{l}$ of nuclease-free water. Mix well by vortexing.

2. Take $1 \mu \mathrm{l}$ of $100 \times$ diluted library from step 1 and dilute it further by adding another $99 \mu$ of nuclease-free water. Mix well by vortexing.

3. Quantify the molar DNA concentration of the $10,000 \times$ diluted samples using the Library Quantification Kit (KAPA Biosystems) and a qPCR thermocycler according to the manufacturer's protocol.

4. Using the concentration determined for the $10,000 \times$ diluted samples, calculate the concentration of the original undiluted libraries from Subheading 3.15.

5. Using the concentrations calculated in step 4, create $20 \mu \mathrm{l}$ diluted RRBS libraries ( $10 \mathrm{nM}$ ) for each original RRBS library. This can be achieved by transferring 0.2 pmol of each library (Subheading 3.14) to separate nuclease-free tubes and topping them up to $20 \mu \mathrm{l}$ with nuclease-free water. 
3.16 qPCR Validation of Knockout Efficiencies
6. Pool $7.5 \mu \mathrm{l}$ of each $10 \mathrm{nM}$ RRBS library into a single $1.5 \mathrm{ml}$ nuclease-free tube to a final volume of $30 \mu \mathrm{l}$.

7. Measure the DNA concentration using Qubit ${ }^{\mathrm{TM}}$ DNA HS Assay Kit.

8. Spike in an amount of the amplicon sequencing libraries (Subheading 3.9) to the multiplexed libraries (step 6), such that each amplicon sequencing library makes up $\sim 0.03 \%$ of the total concentration in ng (see Note 18).

9. Sequence the multiplexed libraries $(10 \mathrm{nM})$ on an Illumina sequencer (HiSeq X or equivalent (see Note 18 ).

To assess the efficiency of tet1/tet2/tet 3 knockout qPCR can be used. In this step, cDNA is created using the SensiFAST ${ }^{\text {TM }}$ cDNA Synthesis kit from both biological replicates of injected and uninjected RNA samples from Subheading 3.8. The cDNA is then used in a standard qPCR experiment to further estimate knockout levels for each tet gene (Fig. lc).

1. Using the SensiFAST ${ }^{\mathrm{Tm}}$ cDNA Synthesis Kit, create cDNA from each RNA sample from Subheading 3.8 by combining $10 \mu l$ nuclease-free water, $5 \mu \mathrm{l}$ RNA, $4 \mu \mathrm{l} 5 \times$ TransAmp buffer, and $1 \mu$ reverse transcriptase in separate $0.2 \mathrm{ml}$ PCR tubes. Mix gently by pipetting.

2. Perform the reverse transcription reaction in a thermocycler.

(a) $10 \mathrm{~min}$ at $25^{\circ} \mathrm{C}$ (annealing).

(b) $15 \mathrm{~min}$ at $42{ }^{\circ} \mathrm{C}$ (reverse transcription).

(c) 5 min at $85^{\circ} \mathrm{C}$ (inactivation).

(d) Hold at $4{ }^{\circ} \mathrm{C}$.

3. Dilute each cDNA sample by adding $80 \mu$ l of nuclease-free water. Mix by pipetting.

4. Create five qPCR master mixes, one for each tet gene and the two control genes, as shown in Table 5 (see Note 19). Mix well by pipetting. Each mix is enough for 14 samples.

5. Add $1 \mu \mathrm{l}$ of each diluted cDNA to 15 wells in a 384 PCR plate, using a different row for each sample, as follows:

(a) tet $1 / 2 / 3 \mathrm{KO}$ replicate 1 to row $\mathrm{A}(\mathrm{Al}-\mathrm{Al} 5)$.

(b) tet $1 / 2 / 3 \mathrm{KO}$ replicate 2 to row $\mathrm{B}(\mathrm{Bl}-\mathrm{Bl} 5)$.

(c) Uninjected control replicate 1 to row $\mathrm{C}(\mathrm{Cl}-\mathrm{Cl} 5)$.

(d) Uninjected control replicate 2 to row D (Dl-Dl5).

6. Add $9 \mu \mathrm{l}$ of each master mix to three columns from A-D as follows:

(a) Master mix 1 to columns $1-3(\mathrm{Al}-\mathrm{A} 3, \mathrm{Bl}-\mathrm{B} 3, \mathrm{Cl}-\mathrm{C} 3$, Dl-D3). 


\section{Table 5}

\section{qPCR master mixes}

\begin{tabular}{llllll}
\hline & Gene & Forward Primer $(\mathbf{1 0} \boldsymbol{\mu M})$ & Reverse Primer $(\mathbf{1 0} \boldsymbol{\mu M})$ & $\mathbf{2} \times$ KAPA Ready Mix & $\mathbf{H}_{\mathbf{2}} \mathbf{0}$ \\
\hline 1 & tet1 & $7 \mu \mathrm{l}$ tet1 qPCR F & $7 \mu \mathrm{l}$ tetl qPCR R & $70 \mu \mathrm{l}$ & $42 \mu \mathrm{l}$ \\
2 & tet 2 & $7 \mu \mathrm{l}$ tet 2 qPCR F & $7 \mu \mathrm{l}$ tet2 qPCR R & $70 \mu \mathrm{l}$ & $42 \mu \mathrm{l}$ \\
3 & tet3 & $7 \mu \mathrm{l}$ tet 3 qPCR F & $7 \mu \mathrm{l}$ tet3 qPCR R & $70 \mu \mathrm{l}$ & $42 \mu \mathrm{l}$ \\
4 & efla & $7 \mu \mathrm{l}$ elfa qPCR F & $7 \mu \mathrm{l}$ elfa qPCR R & $70 \mu \mathrm{l}$ & $42 \mu \mathrm{l}$ \\
\hline 5 & bactin & $7 \mu \mathrm{l}$ bactin qPCR F & $7 \mu \mathrm{l}$ bactin qPCR R & $70 \mu \mathrm{l}$ & $42 \mu \mathrm{l}$ \\
\hline
\end{tabular}

(b) Master mix 2 to columns 4-6 (A4-A6, B4-B6, C4-C6, D4-D6).

(c) Master mix 3 to columns 7-9 (A7-A9, B7-B9, C7-C9, D7-D9).

(d) Master mix 4 to columns 10-12 (Al0-Al2, B10-B12, Cl0-Cl2, Dl0-Dl2).

(e) Master mix 5 to columns 13-15 (Al3-Al5, Bl3-B15, Cl3-C15, Dl3-Dl5).

7. Seal the plate with optically clear sealing tape.

8. Run the plate in a qPCR thermocycler compatible with 384 PCR plates, such as the LightCycler 480 (Roche), under the following conditions:

(a) 5 min at $95^{\circ} \mathrm{C}$.

(b) 35 cycles of $30 \mathrm{~s}$ at $95^{\circ} \mathrm{C}, 45 \mathrm{~s}$ at $60^{\circ} \mathrm{C}$, plate read.

9. Export the CT values from the run into excel sheet in order to calculate the tet $1 / 2 / 3 \mathrm{KO}$ efficiencies. An example of some successful results can be seen in Fig. lc. The values for each tet gene triplicate are calculated individually against the average control CTs, before the average of all these values are plotted with standard deviation. An example of these calculation for tet $I$ replicate $\mathrm{l}$ in tet TKO replicate $\mathrm{l}$ and WT replicate $\mathrm{l}$ are as follows (pay close attention to when replicate CT values are used compared to average values, as well as when WT values are used):

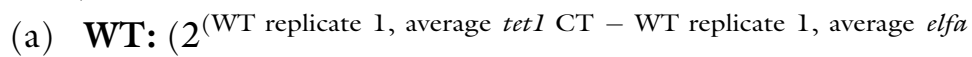
$\mathrm{CT}) /\left(2^{\text {(WT replicate } 1 \text {, tet } 1 \text { replicate } 1 \mathrm{CT}-\text { WT replicate } 1, \text { elfa } \mathrm{CT})}\right)$ and $(2$ (WT replicate 1 , average tet 1 CT - WT replicate 1 , average bactin

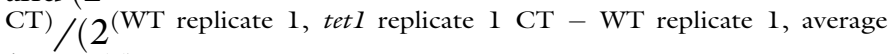
bactin $\mathrm{CT})$.

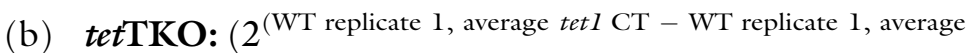

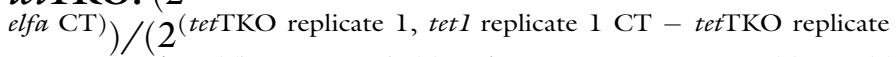

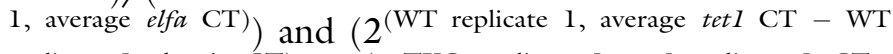

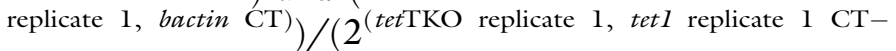
tet $\mathrm{TKO}$ replicate $\mathrm{l}$, average bactin $\mathrm{CT})$. 
3.17 Software

3.18 File Names
Download the following software:

1. FastQC http://www.bioinformatics.babraham.ac.uk/pro jects/fastqc/

2. Trim Galore https://www.bioinformatics.babraham.ac.uk/pro jects/trim_galore/

3. SAMtools http://samtools.sourceforge.net/ [23].

4. Sambamba https://lomereiter.github.io/sambamba/ [24].

5. WALT https://github.com/smithlabcode/walt [25].

6. MethylDackel https://github.com/dpryan79/MethylDackel

7. BEDtools https://bedtools.readthedocs.io/en/latest/ [26].

8. bedGraphToBigWig, fetchChromSizes, and liftOver http:// hgdownload.cse.ucsc.edu/admin/exe

9. IGV http://www.broadinstitute.org/igv/ [27].

10. CRISPResso https://github.com/lucapinello/ CRISPResso [28].

1. In the following pipeline, paired FASTQ files generated from RRBS and amplicon sequencing should be named as follows (see Note 20):

(a) tet $1 / 2 / 3 \mathrm{KO}$ replicate 1 Read I: tetTKO_1_Rl.fastq.gz.

(b) tet $1 / 2 / 3 \mathrm{KO}$ replicate 1 Read 2: tetTKO_1_R2.fastq.gz.

(c) tet $1 / 2 / 3 \mathrm{KO}$ replicate $2 \mathrm{Read} 1$ : tetTKO_2_Rl.fastq.gz.

(d) tet 1/2/3 KO replicate 2 Read 2: tetTKO_2_R2.fastq.gz.

(e) Uninjected replicate 1 Read 1: WT_1_Rl.fastq.gz.

(f) Uninjected replicate 1 Read 2: WT_1_R2.fastq.gz.

(g) Uninjected replicate 2 Read 1: WT_2_Rl.fastq.gz.

(h) Uninjected replicate 2 Read 2:WT_2_R2.fastq.gz.

(i) Amplicon sequencing library for tet $1 / 2 / 3 \mathrm{KO}$ replicate 1 Read 1: Amp_1_Rl.fastq.gz.

(j) Amplicon sequencing library for tet $1 / 2 / 3 \mathrm{KO}$ replicate 1 Read 2: Amp_1_R2.fastq.gz.

(k) Amplicon sequencing library for tet $1 / 2 / 3 \mathrm{KO}$ replicate 2 Read 1: Amp_2_Rl.fastq.gz

(1) Amplicon sequencing library for tet $1 / 2 / 3 \mathrm{KO}$ replicate 2 Read 2: Amp_2_R2.fastq.gz.

2. Files can be renamed using the $(\mathrm{mv})$ command as follows:

\$ mv OriginalName.ext NewName.ext 


\subsection{Quality Control}

\subsection{Adapter} Trimming
1. If the FASTQ files were delivered without accompanying FastQC files (see Note 21), a quality report can be created for all FASTQ files as follows:

\$astqc *.fastq.gz

2. There are a number of hallmark features of a successful RRBS experiment to assess before performing quality trimming, such as: (i) a high level of duplication and (ii) read 1 sequences beginning with CGG ( see Note 20). An example of these features can be seen in Fig. 2.

1. Quality-trim and remove any adapter sequences from the paired tetTKO_l FASTQ files using Trim Galore in RRBS mode (see Note 22).

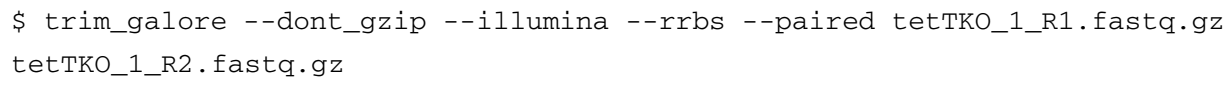

Repeat for the rest of the paired FASTQ files. The trimmed FASTQ files will be produced with the suffixes "_val_l.fq" and “_val_2.fq" for Read 1 and Read 2, respectively. For example: tetTKO_1_R1_val_1.fq and tetTKO_1_R2_val_2.fq will be produced from the above command.

A

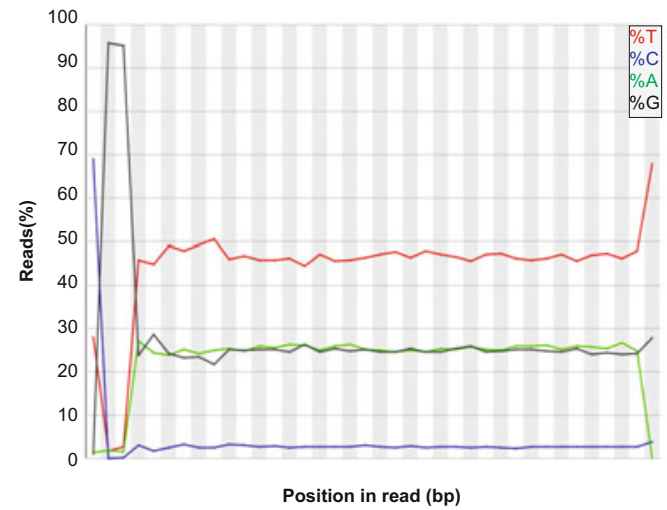

B

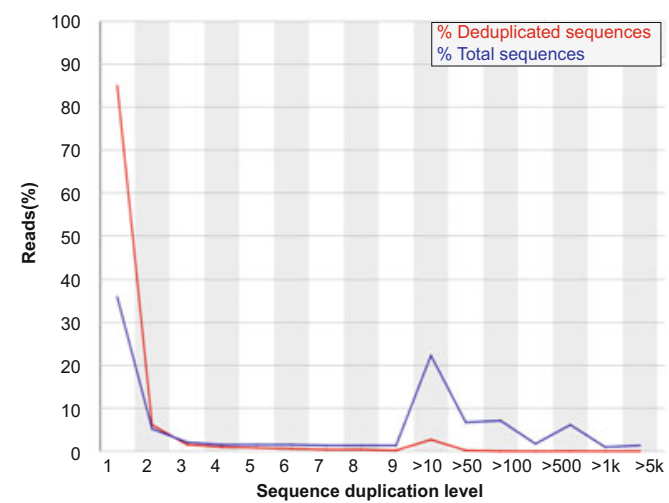

Fig. 2 Quality assessment of RRBS sequence data. (a) Sequence content across all bases from the FASTQ file for read 1 showing enrichment for CGG or TGG trinucleotides at the beginning of read 1. This bias is due to Mspl digestion, which creates fragments beginning with CGG nucleotides (methylated state) or TGG (unmethylated state). (b) Sequence duplication level of the FASTQ file for Read 1. This is another hallmark of successful Mspl digestion as each DNA molecule should produce identical fragments 
3.21 Amplicon Sequencing Analysis
1. Use CRISPResso to determine the frequencies of non-homologous end joining (NHEJ) events for each tet gene in the amplicon sequencing library for tet $1 / 2 / 3 \mathrm{KO}$ replicate 1 (Amp_1_Rl_val_1.fq; Amp_1_R2_val_2.fq). CRISPResso will generate a number of informative graphs and reports (see Note $\mathbf{2 3}$ ).

\$ CRISPResso -r1 Amp_1_R1_val_1.fq -r2 Amp_1_R2_val_2.fq -a C ATTCCCACACTCTGTCCGTATGTTACTTGTGACTCACTCCTCATTGAGGGCA CAGCGTCGGCTAGTGGGCGAGCCATATTTGCAGAGCGTCTGCGTGAGCTCCTGGTA CAGCCTGTCAGCCACGCTGCGTGGGATCCCCTCCCAGGCCAAGATGAGGATGACTAT

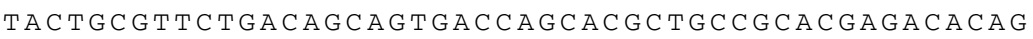
CAACTTCTCCTGCTCACTGCCTCGACGTATGA -g GgGCACAGCGTCGGCTAGT, G GAGGGGATCCCACGCAGCG, A GAAGTTGCTGTGTCTCGTG --ignore_substitu tions -w 10 -o tet1_rep1

\$ CRISPResso -r1 Amp_1_R1_val_1.fq -r2 Amp_1_R2_val_2.fq -a A GACTTGTTGTCTTGATCATTGTTTCGATTTTGAGAAGGGGAAGTACATGCCTGGGGAAT CAACTTGCTCTCTACTGCTTGTGGCTCATGAGTCTGTTGCTGAAGCAAGGAAAAGTCTC TATTGCCATGGAGGTTTTCATCAGTTGTTTTTTTCTGTGGCACTTTAATCTGTGGGTTA TACTGACCTGTCTGAGAGGTTTGTGAGGGGGAACCTGCTGTCACCGGGGGCAATGTATAA

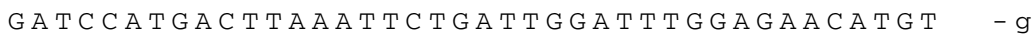
AGAAGGGGAAGTACATGCCT, AGGAAAAGTCTCTATTGCCATGG, GAGGGGGAACCTGCTGT CAC --ignore_substitutions -w 10 -o tet2_rep1

\$ CRISPResSo -r1 Amp_1_R1_val_1.fq -r2 Amp_1_R2_val_2.fq -a T TTTTGGCCTCCTCACCTCCATTGGACATTTCCTGATTGAATCTTTCATACAGCTGAGCTG TAGGGGTCGGTGAAGGTTGGTGGTTGGTGTAAGGTGGCAGTGAGCCAACAGAAAAGGCCAC CAGAGTTTGTGCATCCTCCATATCCACATCATTTACTGTTGTGCTGGCATTTTGACCACTC CAAACACCTTGTTGAGATTCAGGATTTGACAACTTCCCAGTATGCTCAAGGACC

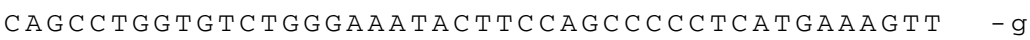

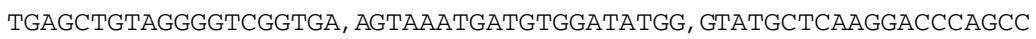
--ignore_substitutions -w 10 -o tet3_rep1

2. Repeat for the amplicon sequencing library for tetl/2/3 $\mathrm{KO}$ replicate 2 (Amp_2_Rl_val_1.fq; Amp_2_R2_val_1.fq). An example of CRISPResso outputs from a successful tet $1 / 2 / 3$ $\mathrm{KO}$ experiment can be seen in Fig. 3.

3. Amplicon sequencing results corresponding to RRBS data are outlined in Table 6.

\subsection{Read Mapping}

1. Download the zebrafish reference genome, danRer10, from UCSC.

\$ wget http://hgdownload.soe.ucsc.edu/ goldenPath/danRer10/bigzips/danRer10.fa.gz \$ gunzip danRer10.fa.gz 
A

A

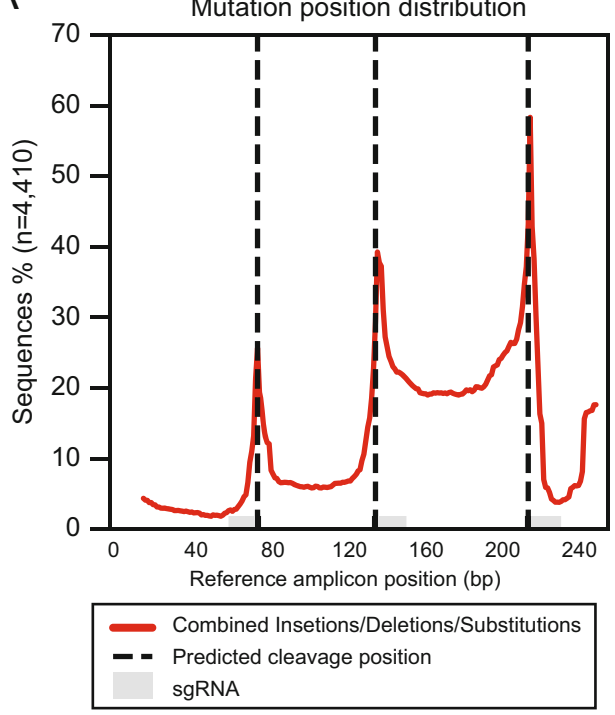

B

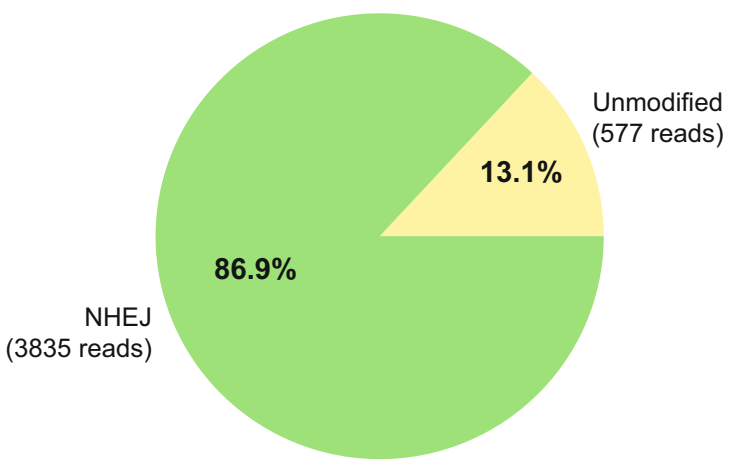

C

TGACCAGCACGCTGCCGCACG'AGACACAGCAACT T C T C C T Reference

T GA C C A G C A C G C T G C C G C A C G A G A C A C A G C A A C T T C T C C T 21.24\% (937 reads) T G A C C A G C A C G C T G C C G C A I A A A A G C A A C T T C T C C T $6.87 \%$ (303 reads)

T G A C C A G C A C G C T G C C G C A C I - A C A G C A A C T T C T C C T $4.42 \%$ (195 reads) GIA G A C A C A G C A A C T T C T C C T $3.58 \%$ (158 reads) - - - A C A C A G C A A C T T C T C C T 2.54\% (112 reads) T GAC CAGCACGCTGCCG_... C A C A G C A A C T T C T C C T $2.43 \%$ (1-7 reads) . T G A C C A G C A C G C T G C C G C - - - - A C A C A G C A A C T T C T C C T $1.68 \%(74$ reads) - - - - - - - - - - - - - - -

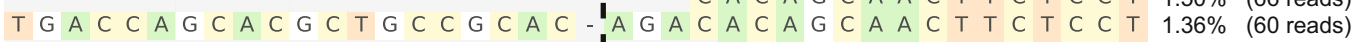

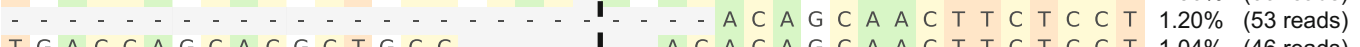
T G A C C A G C A C G C T G C C - . - . - A C A C A G C A A C T T C T C C T 1.04\% (46 reads)

- Deletions - Predicted cleavage position

Fig. 3 Analysis of genome editing efficiency of tet 1 locus from the tet1/2/3 $\mathrm{K} 0$ amplicon sequencing. (a) Locations of insertions and deletions surrounding the targeted tet 1 locus. (b) Quantification of unmodified and non-homologous end joining (NHEJ) events in the tet1 amplicon library. (c) Alleles surrounding the cut site for one of the tet $1 \mathrm{gRNAs}$

Table 6

Amplicon sequencing results corresponding to RRBS libraries

\begin{tabular}{llll}
\hline Sample & tet1 NHEJ & tet2 NHEJ & tet3 NHEJ \\
\hline tet1/2/3 KO replicate 1 & $86.9 \%$ & $64.3 \%$ & $97.8 \%$ \\
\hline tet1/2/3 KO replicate 2 & $94.2 \%$ & $41.5 \%$ & $93.2 \%$ \\
\hline
\end{tabular}


2. Download the lambda reference genome and annotate it as chrL (see Note 24).

\$ wget -o lambda.txt.gz 'https://www. encodeproject.org/files/lambda.fa/@adownload/

lambda.fa.fasta.gz'

\$ gunzip lambda.txt.gz

\$fold-w 50 lambda.txt | sed'\$d'>lambda.fa

3. Concatenate the lambda genome to the zebrafish genome.

\$ cat danRer10.fa lambda.fa > danRer10_lambda.fa

4. Index the combined genome using WALT.

\$ makedb -c danRer10_lambda.fa -o danRer10_lambda.dbindex

5. Map the trimmed paired FASTQ files for the tet $1 / 2 / 3 \mathrm{KO}$ replicate 1 library (tetTKO_1_R1_val_1.fq; tetTKO_1_R2_val_2.fq) to the reference genome using WALT. To speed up the process of mapping, and many other steps in this pipeline, multiple threads can be used for many such commands ( see Note 25). For example, in the below command and the commands in step 7, 12 threads $(-t)$ are used.

\$ walt -i danRer10_lambda.dbindex -1 tetTKO_1_R1_val_1.fq -2 tetTKO_1_R2_val_2.fq -o tetTKO_1.sam -t 12

6. Repeat with the trimmed paired FASTQ files for tetTKO_2, WT_1 and WT_2, remembering to also change the output name (-o) accordingly.

7. Convert the tetTKO_1.sam SAM file to a sorted BAM file (see Note 26).

\$ sambamba view -t 12 -S -f bam -h tetTKo_1.sam | sambamba sort -t 12 -o tetTKO_1.bam /dev/stdin

8. Repeat with the tetTKO_2.bam, WT_1.bam, and WT_2.bam files, remembering to also change the output name $(-0)$ accordingly.

3.23 Methylation

Calling
1. Check the methylation bias for the tetTKO_l.bam file (see Note 27).

\$ MethylDackel mbias danRer10_lambda.fa tetTKO_1.bam tetTKO_1 
2. Repeat with the tetTKO_2.bam, WT_1.bam, and WT_2.bam files, remembering to also change the output name prefix accordingly.

3. Using MethylDackel, call methylation levels for CG dinucleotides from the tetTKO_l.bam file, correcting for genome variation and methylation bias (see Notes 27 and 28). This command will create a bedGraph file (see Note 29).

\$ MethylDackel extract --mergeContext --maxVariantFrac 0.25 --

minoppositeDepth 2 --OB 10,140,10,140 --OT 10,140,10,140

danRer10_lambda.fa tetTKO_1.bam

4. Repeat for the tetTKO_2.bam, WT_1.bam, and WT_2.bam files.

5. Methylation levels can also be called for all $\mathrm{C}$ nucleotide contexts if desired ( see Note 30 ).

6. Remove the header from each CG bedGraph file (see Note 31 ).

$\$$ sed $-i{ }^{\prime} 1 d^{\prime} *$ bedGraph

7. Estimate the CG methylation non-conversion percentage by calculating the methylation values assigned to the unmethylated lambda control from the tetTKO_1_CpG.bedGraph (see Note 24).

$\$$ grep chrL tetTKO_1_Cpg.bedGraph | awk $\quad\{\mathrm{s}+=\$ 5\}\{\mathrm{x}+=\$ 6\}$ END \{print $\mathrm{s} /(\mathrm{x}+\mathrm{s}) * 100\}^{\prime}$

8. Repeat for tetTKO_2_CpG.bedGraph, WT_1_CpG.bedGraph, and WT_2_CpG.bedGraph files. The output value from the above command is given as a percentage.

9. Non-conversion rates can also be calculated for $\mathrm{CHG}$ and $\mathrm{CHH}$ nucleotides from their corresponding bedGraphs if desired.

1. Download supplementary Tables 6-20 from https://www. nature.com/articles/ng.3522\#Sec29 [12]. Copy and merge supplementary Tables 8 and 10, without the headers, into a new excel document on the same sheet. Save the new document as a tab-delimited file with the name phyloDMRs_danRer7.bed (see Note 32). This BED file now contains the coordinates of all the regions that become developmentally hypomethylated after gastrulation in a Tet-dependent manner in zebrafish.

2. Transfer this file into the directory of the CpG bedGraphs.

3. Lift over the coordinates of the phyloDMRs from danRer7 to danRer10 using liftOver (see Note 33). 
\$ wget https://hgdownload.soe.ucsc.edu/gbdb/ danRer 7 /liftover/danRer 7ToDanRer10. over.chain. gz

\$ liftover phyloDMRs_danRer7.bed danRer7To DanRer10.over.chain.gz phyloDMRs.bed unmapped. bed

4. Sort the phyloDMRs.bed BED file.

\$ sort -k1,1 -k2,2n -o phyloDMRs.bed phyloDMRs.bed

5. Sort the tetTKO_1_CpG.bedGraph file.

\$ sort -k1,1 -k2,2n -o tetTKO_1_Cpg.bedGraph tetTKO_1_Cpg. bedGraph

6. Repeat for the tetTKO_2_CpG.bedGraph, WT_1_CpG.bedGraph, and WT_2_CpG.bedGraph files.

7. Create a 4 column bedGraph file from the sorted tetTKO_1_CpG.bedGraph file.

\$ awk ' OFS=" $\backslash t "$ " $\{$ print $\$ 1, \$ 2, \$ 3, \$ 4 / 100\}$ ' tetTKO_1_CpG.

bedGraph | sed '/^ChrL/d'> tetTKO_1_CpG_4col.bedGraph

8. Repeat for the tetTKO_2_CpG.bedGraph, WT_1_CpG.bedGraph, and WT_2_CpG.bedGraph files.

9. Download a chromosome size file for danRerl0.

\$ fetchChromsizes danRer10 > chrom.sizes

10. Convert the tetTKO_1_CpG_4col.bedGraph file to a BigWig file (see Note 34).

\$ bedGraphToBigWig tetTKO_1_CpG_4col.bedGraph chrom.sizes tetTKO_1_CpG.bw

11. Repeat for the tetTKO_2_CpG_4col.bedGraph, WT_1_CpG_4col.bedGraph, and WT_2_CpG_4col.bedGraph files.

12. All BAM files, Bigwig files, and the phyloDMRs.bed file can now be loaded into the IGV browser for visualization ( see Note 35). An example of both the BAM file and Bigwig file outputs can be seen in Fig. 4 a.

3.25 Methylation Phenotype Analysis
To interrogate the methylation phenotypes of the tet $1 / 2 / 3 \mathrm{KO}$ samples, average methylation levels for phyloDMRs, and MspI fragments, can be determined. 
A

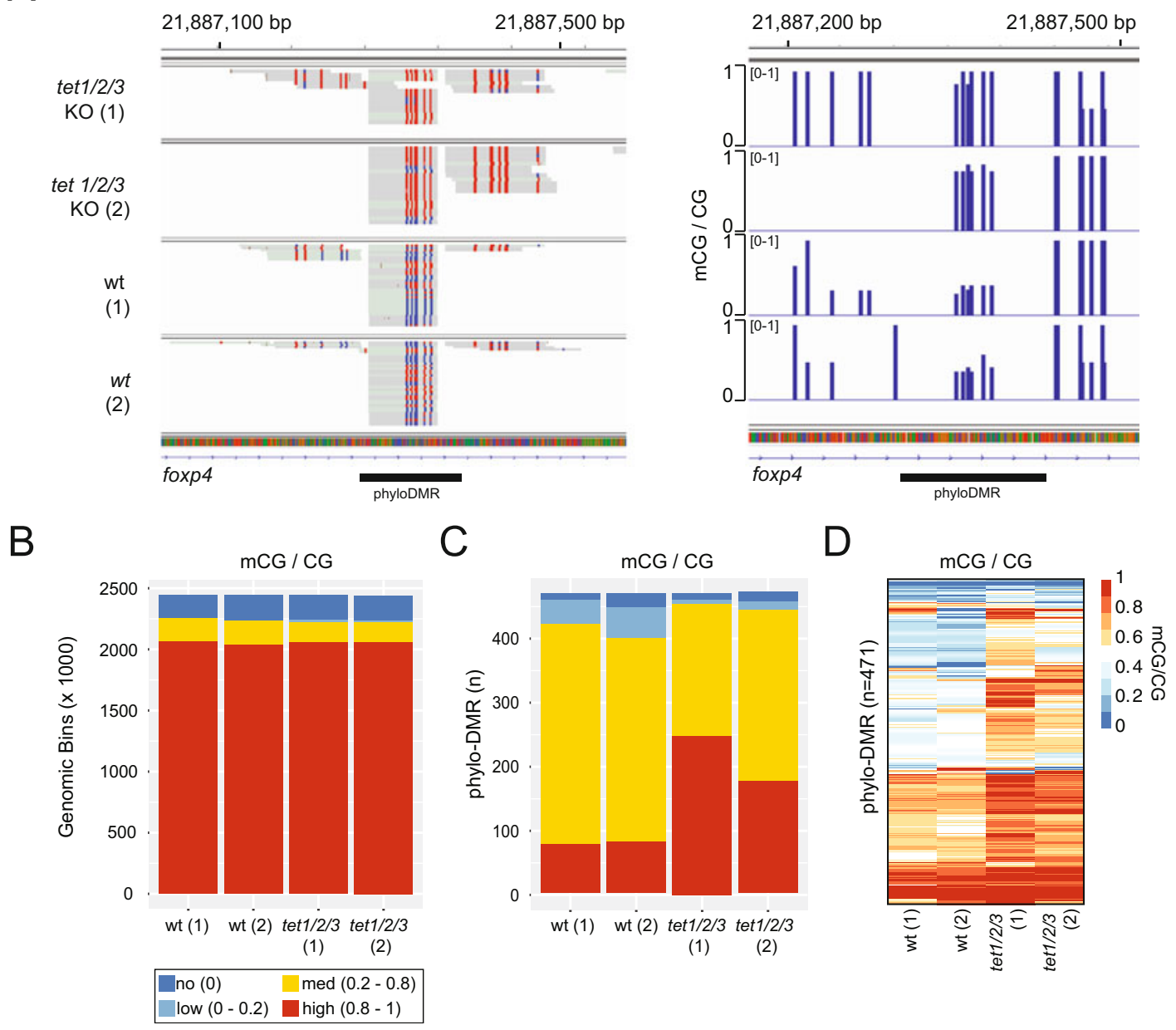

Fig. 4 Visualization and analysis of tet1/2/3 KO RRBS data. (a) Left panel: IGV visualization of BAM files in bisulfite mode (CG) over a phylo-DMR (phylotypic stage enhancer) region. Red = methylated cytosine, blue $=$ unmethylated cytosine. Grey boxes represent single reads. Right panel: IGV visualization of BigWig files. Blue bars represent methylation level (mCG/CG). (b) Global mCG/CG levels of two biological replicates of uninjected (wt) and injected (tet1/2/3 K0) 24hpf F0 zebrafish. Methylation values correspond to average $\mathrm{mCG} / \mathrm{CG}$ level in genomic bins (150 bp). (c) PhyloDMR mCG/CG levels of two biological replicates of uninjected (wt) and injected (tet1/2/3 K0) $24 \mathrm{hpf}$ F0 zebrafish. (d) Heatmap showing locus-specific mCG/CG levels in phyloDMRs

1. Using bedtools, create a BED file with the phyloDMRs that are covered in all the bedGraphs (see Note 36).

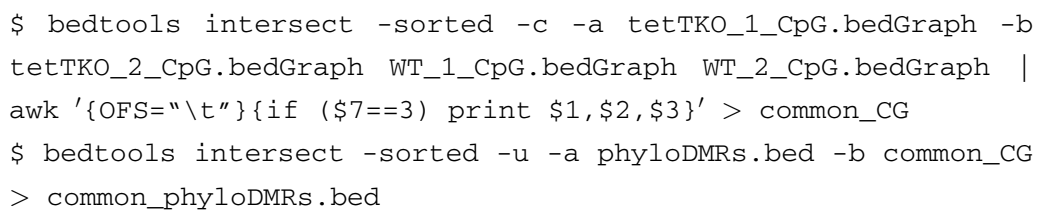


2. Calculate the average methylation levels across the commonly covered phyloDMRs for the tetTKO_1_CpG.bedGraph.

\$ bedtools map -a common_phyloDMRs.bed -b tetTKO_1_CpG.

bedGraph -C 5,6 -o sum, sum | awk ' \{print $\$ 4 /(\$ 4+\$ 5)\}^{\prime}>$ tetTKO_1_phyloDMRs_mCG-CG

3. Repeat for the tetTKO_2_CpG.bedGraph, WT_1_CpG.bedGraph, and WT_2_CpG.bedGraph files.

4. Using bedtools, merge all commonly covered CG dinucleotides into windows that loosely represent MspI fragments (see Note 37).

\$ bedtools merge -d 150 -i common_CG > common_CG_windows

5. Calculate the average methylation for these CG windows for the tetTKO_1_CpG.bedGraph.

\$ bedtools map -a common_CG_windows -b tetTKO_1_CpG.bedGraph -C 5,6 -o sum, sum | awk '\{print $\$ 4 /(\$ 4+\$ 5)\}^{\prime}>$ tetTKO_1_

common_mCG-CG

6. Repeat for the tetTKO_2_CpG.bedGraph, WT_1_CpG.bedGraph, and WT_2_CpG.bedGraph files.

7. Create tables, which can be easily analyzed in $\mathrm{R}$, for both the average methylation of phyloDMRs and the average methylation of these common MspI CG windows.

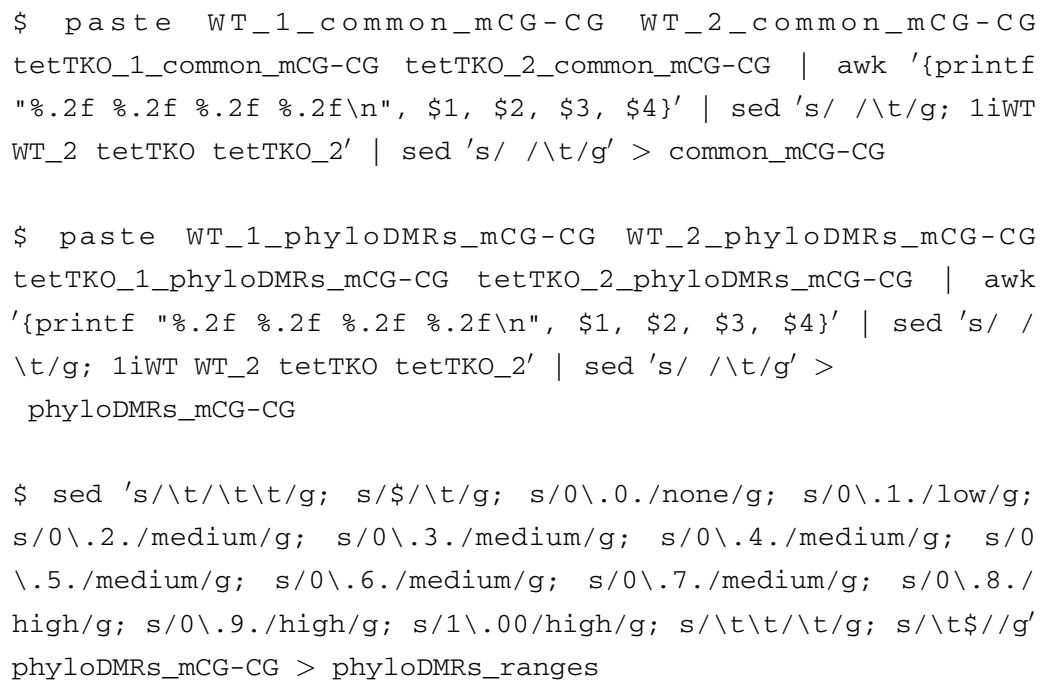


$\$ \mathrm{sed} ' \mathrm{~s} / \mathrm{t} / \backslash \mathrm{t} \backslash \mathrm{t} / \mathrm{g} ; \mathrm{s} / \$ / \backslash t / \mathrm{g} ; \mathrm{s} / 0 \backslash .0 . / \mathrm{none} / \mathrm{g} ; \mathrm{s} / 0 \backslash .1 . / 1 \mathrm{ow} / \mathrm{g} ;$ $\mathrm{s} / 0 \backslash .2 . /$ medium/g; s/0 $\backslash .3 . /$ medium/g; s/0 $\backslash .4 . /$ medium/g; s/0

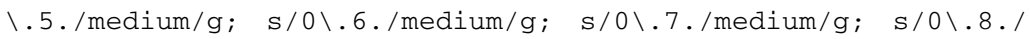

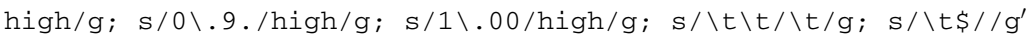
common_mCG-CG > common_ranges

8. In $\mathrm{R}$, create stacked plots showing the average methylation values for each sample at phyloDMRs and for the common MspI CG windows (Fig. 4b,c).

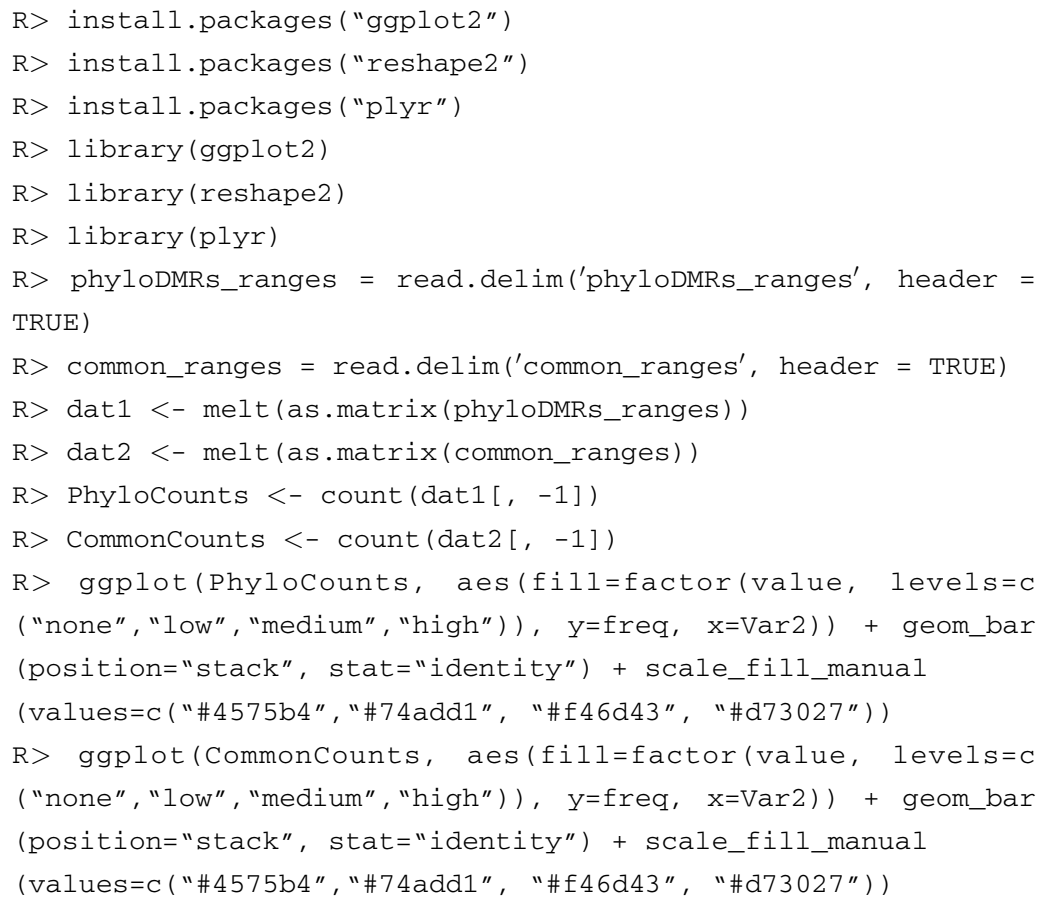

9. Methylation levels at each locus can be visualized using pheatmaps in R (Fig. 4d) (see Note 38).

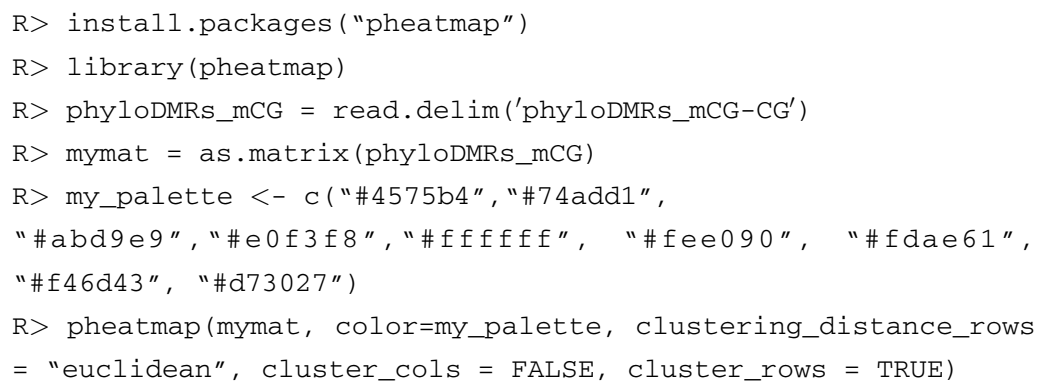

10. An example of plots from a successful tetl/2/3 KO RRBS experiment can be seen in Fig. 4 . 


\section{Notes}

1. The protospacer sequence is the genomic sequence that is targeted by the gRNA and is thus complementary to a portion of the gRNA. When using the Cas9 nuclease, the protospacer sequence must be followed by the protospacer adjacent motif (PAM): NGG. Cas9 will cut the protospacer sequence 3 base pairs away from the PAM site.

2. AMPure XP beads should be regularly tested by performing purifications on DNA ladders using different ratios of beads to ladder. The products can be analyzed by gel electrophoresis to determine the relationships between bead ratios and fragment size selection.

3. The supernatant from the AMPure XP beads can be most effectively removed with multiple rounds of pipetting with short tabletop centrifugation in between each attempt.

4. When eluting, it is recommended to add an extra $1-2 \mu l$ of solution to the volume that will ultimately be taken. This will avoid carrying over any AMPure XP beads into subsequent steps.

5. The gRNA produced by the in vitro transcription will often be visible as a white precipitate. The precipitate can settle to the bottom of tubes so take care to not to leave the gRNA behind when transferring between tubes. This can be achieved by mixing well before any transfers.

6. If the gRNA pellet is not disturbed, simply remove the ethanol with a pipette to complete the wash. If the pellet becomes dislodged, centrifuge for another $5 \mathrm{~min}$ at $4{ }^{\circ} \mathrm{C}$ before removing the ethanol.

7. Air bubbles will often become trapped under the injection mold. Gently tap the mold to try and displace the formed bubbles. The bubbles themselves will not cause any issues if they are smaller than a single egg.

8. While more zebrafish can be added per breeding tank to maximize the amount of eggs, this will result in greater genetic variability in the experiment. To overcome this, use the same fish to lay multiple rounds of eggs in a single day.

9. From each injection 80 embryos will be collected. However, it is best to inject as many eggs as possible as many will be unfertilized, dead or will not display any phenotype.

10. Microinjection of multiple gRNA and Cas9 into the single cell or the yolk have been shown to have comparable results [29]. 
11. The microinjection needle can become clogged during the injection process. To unclog the needle, the pressure can be temporarily increased or the needle can be cut again or replaced.

12. At 24 hpf the tetl $/ 2 / 3 \mathrm{KO}$ phenotypes tend to be very subtle. On average, for a successful injection experiment, approximately $50 \%$ of embryos will display developmental phenotypes as described above. We do not observe any noticeable lethality before 48 hpf compared to wild type controls. However, by $\sim 7$ dpf, nearly all larvae which had phenotypes will die. It is therefore recommended to keep some of the fish until after $48 \mathrm{hpf}$ to get a better estimation of how successful the injection experiment was.

13. RRBS libraries are dependent on high quality gDNA because of their reliance on size selection and restriction enzymes. Any degraded DNA products can be carried over into the final libraries if they are of the right size. The more degraded the gDNA the less enriched the libraries will be.

14. While methylated adapters are not required for amplicon sequencing, we use methylated adapters in this case as excess is available from the kit required for RRBS experiments.

15. The RRBS protocol is optimized for gDNA input of $500 \mathrm{ng}$, however, as little as $100 \mathrm{ng}$ per sample can be used. Libraries that will be compared against each other should start with the same amount of input gDNA.

16. For low level multiplexing, certain barcode indexes need to be used in combination. Follow the manufacturer's instructions from the NEXTflex bisulfite-seq barcodes kit to determine which adapter barcodes to use when multiplexing a different amount of samples.

17. Expected fragment sizes for this protocol range from $250 \mathrm{bp}$ to $500 \mathrm{bp}$, with $\sim 120 \mathrm{bp}$ of each fragment being contributed to by the adapter sequence. The lower limit is determined by the AMPure XP bead size selections while the upper limit is driven by the sodium bisulfite treatment and the preferential amplification of smaller fragments in PCR reactions. A sharp band can sometimes appear at $\sim 120$ bp and this is caused by adapter dimer contamination. If this band is present, repurify the libraries as in Subheading 3.12.

18. ROX is a reference dye which is used to normalize the fluorescent reporter signal in some qPCR instruments. $0.2 \mu \mathrm{l}$ low ROX or $0.2 \mu \mathrm{l}$ high ROX may be added to each sample depending on the instrument used.

19. A single lane of the HiSeq $X$ yields approximately $2 \times 400$ million, 150 bp paired end reads. $2 \times 20$ million paired end 
reads are sufficient for an RRBS library using zebrafish gDNA. Zebrafish bisulfite-based libraries most commonly map to the genome with $\sim 60 \%$ mapping efficiency which will yield approximately $2 \times 12$ million usable paired end reads. In this protocol, as only 4 RRBS libraries are pooled together, only $10 \%$ of a single lane is needed. Up to 20 RRBS libraries can be pooled into a single HiSeq X lane. Each amplicon from an amplicon sequencing library requires $5-10 \mathrm{k}$ reads, which is the equivalent of $\sim 0.01 \%$ of what is required for four RRBS libraries (or $0.03 \%$ for a pool of 3 amplicons).

20. The data from Illumina sequencer is usually converted into FASTQ format [30]. The index sequence from each adapter barcode allows the sequencing data to be de-multiplexed into multiple FASTQ files, which contain both the sequence and quality information for each library. In paired end sequencing, separate FASTQ files for Read 1 and Read 2 will be generated for each library.

21. The quality of FASTQ files for each library can be assessed with FastQC. FastQC produces a number of informative graphs and tables relating to both sequence quality and content. There are some hallmark features of a FastQC report from a successful RRBS library. The "Per base sequence content" should show that nearly $100 \%$ of reads begin with a $\mathrm{C}$ or $\mathrm{T}$ followed by GG. This is due to the MspI digestion creating fragments beginning with CGG nucleotides. These will remain as CGG if methylated or become TGG if they were unmethylated. RRBS libraries should also have a high level of duplication, on average greater than $70 \%$ duplication rate, as every copy of DNA should produce the same fragments (Fig. 2). Therefore, unlike in WGBS, deduplication of the data is not recommended.

22. RRBS libraries created with MspI digestion need to have 2 bp from the $3^{\prime}$ end of Read 1 removed and the first 2 bp of Read 2 removed. This is because MspI digestion causes overhangs which get filled in during the end repair process. This means an unmethylated cytosine will always be introduced in the fragment regardless of the original methylation state.

23. CRISPResso is used to analyze amplicon sequencing libraries and determine the efficiency of tet $1 / 2 / 3 \mathrm{KO}$. CRISPResso generates graphs and tables relating to the percentage of reads with detectable NHEJ events, as well as the position and sequence of these events. As zebrafish display considerable genetic diversity, CRISPResso is best used with substitutions ignored (--ignore_substitutions) to avoid calling NHEJ events which are merely polymorphisms. A window of $10 \mathrm{bp}$ around the cut site (-w 10) is also used to avoid including errors from the start and end of reads. 
24. The lambda genome is used as a control for testing the non-conversion rate of the libraries. As unmethylated lambda DNA was spiked into each library, the non-conversion rate can be determined by the percentage of cytosines which remained unconverted after the bisulfite conversion process.

25. Multiple threads, and in some instances cores, can be allocated to running commands to greatly speed up the time taken for completion. To find out if commands have the options for allocating multiple threads/cores, it is best to read the manuals which come when installing the commands or to visit their associated webpages.

26. SAM files are text-based files which contain the alignment information and read information generated by mapping the FASTQ files [23]. SAM files can be converted into a compressed binary format called a BAM file.

27. RRBS libraries are prone to methylation biases across the length of the read. It is highly recommended to use MethylDackel to analyze this (mbias) and then correct for it in the subsequent methylation calling steps.

28. As zebrafish are highly polymorphic, it is important to perform a genome correction to avoid erroneously calling genomic sequence changes as methylation changes.

29. BedGraph files are similar to BED files ( see Note 32); however, they also must have a fourth column with a value. In this case, this value is the methylation percentage. BedGraphs produced by MethylDackel also have additional fifth and sixth columns, and these are the number of reads reporting a methylated base and unmethylated base, respectively.

30. Zebrafish display both $\mathrm{mCG}$ an $\mathrm{mCH}$ methylation dynamics during embryogenesis [31]. One can thus extend the analyses to all $\mathrm{C}$ nucleotides by using the $(-\mathrm{CHH})$ and $(-\mathrm{CHG})$ options in the MethylDackel extract command.

\$ MethylDackel extract --CHH --CHG --maxVariantFrac 0.25 -minDepth 5

--ов 10,140,10,140 --OT 10,140,10,140 danRer10_lambda.fa

tetTKo_1.bam

31. A header left in the bedGraph file can cause some commands to fail, such as BEDtools and liftOver commands.

32. BED files are tab-delimited files that contain 3 columns describing genomic coordinates. BED files contain a chromosome column, start column and end column.

33. The coordinates of phylo-DMRs were originally determined using the danRer7 genome assembly. In order to use them in 
this pipeline, the coordinates must be converted to the danRer10 assembly using the liftOver command.

34. BigWig files are compressed, indexed, and in binary format. They contain signal data, in this case methylation values. BigWig files can be used to visualize data, such as in genome browsers or to create heatmaps.

35. IGV browser can display BED, BedGraph, BAM, and BigWig files. Visualizing BAM files is useful as this type of visualization allows inspection of single reads, thus providing information about the heterogeneity of DNA methylation at specific loci. BigWig files are useful as they show the overall average methylation values at specific loci (Fig. 4).

36. Using only common phylo-DMRs makes comparisons between libraries more valid. It also makes $\mathrm{R}$ commands simpler and heatmaps easier to interpret.

37. RRBS libraries cannot accurately determine global methylation levels due to libraries only covering a fraction of the genome with specific sequence biases. However, using all the regions covered can still be useful in showing that the methylation changes in tet $1 / 2 / 3 \mathrm{KO}$ are more pronounced at phylo-DMR regions. Random control regions can also be created by using BEDtools shuffle.

\$ bedtools shuffle -i phyloDMRs.bed -g chrom.sizes > shuffled_control.bed

38. Heatmaps can also be created by using a variety of other methods such as deepTools (https://github.com/deeptools/ ) and the gplots package in $\mathrm{R}$ (http://cran.r-project.org/ package $=$ gplots $)$.

\section{Acknowledgments}

\section{Australian Research Council (ARC) Discovery Project (DP190103852) to OB supported this work. We thank Michael Geng for the management and breeding of zebrafish colonies.}

\section{References}

1. Angeloni A, Bogdanovic O (2019) Enhancer DNA methylation: implications for gene regulation. Essays Biochem 63:707-715

2. Greenberg MVC, Bourc'his D (2019) The diverse roles of DNA methylation in mammalian development and disease. Nat Rev Mol Cell Biol 20:590-607

3. Hermann A, Goyal R, Jeltsch A (2004) The Dnmtl DNA-(cytosine-C5)-methyltransferase methylates DNA processively with high preference for hemimethylated target sites. J Biol Chem 279:48350-48359

4. Okano M, Bell DW, Haber DA et al (1999) DNA methyltransferases Dnmt3a and Dnmt3b are essential for de novo methylation and mammalian development. Cell 99:247-257

5. Ito S, D'Alessio AC, Taranova OV et al (2010) Role of Tet proteins in $5 \mathrm{mC}$ to $5 \mathrm{hmC}$ 
conversion, ES-cell self-renewal and inner cell mass specification. Nature 466:1129-1133

6. Ito S, Shen L, Dai Q et al (2011) Tet proteins can convert 5-methylcytosine to 5-formylcytosine and 5-carboxylcytosine. Science 333:1300-1303

7. Almeida RD, Loose M, Sottile V et al (2012) 5-hydroxymethyl-cytosine enrichment of non-committed cells is not a universal feature of vertebrate development. Epigenetics 7:383-389

8. Maiti A, Drohat AC (2011) Thymine DNA glycosylase can rapidly excise 5-formylcytosine and 5-carboxylcytosine: potential implications for active demethylation of $\mathrm{CpG}$ sites. J Biol Chem 286:35334-35338

9. Wossidlo M, Nakamura T, Lepikhov K et al (2011) 5-Hydroxymethylcytosine in the mammalian zygote is linked with epigenetic reprogramming. Nat Commun 2:241

10. Ross SE, Bogdanovic O (2019) TET enzymes, DNA demethylation and pluripotency. Biochem Soc Trans 47:875-885

11. Dai HQ, Wang BA, Yang L et al (2016) TET-mediated DNA demethylation controls gastrulation by regulating lefty-nodal signalling. Nature 538:528-532

12. Bogdanovic O, Smits AH, de la Calle Mustienes E et al (2016) Active DNA demethylation at enhancers during the vertebrate phylotypic period. Nat Genet 48:417-426

13. Li C, Lan Y, Schwartz-Orbach L et al (2015) Overlapping requirements for Tet 2 and Tet 3 in Normal development and hematopoietic stem cell emergence. Cell Rep 12:1133-1143

14. Domazet-Lošo T, Tautz D (2010) A phylogenetically based transcriptome age index mirrors ontogenetic divergence patterns. Nature 468:815-818

15. Hsu PD, Lander ES, Zhang F (2014) Development and applications of CRISPR-Cas9 for genome engineering. Cell 157:1262-1278

16. Meissner A, Gnirke A, Bell GW et al (2005) Reduced representation bisulfite sequencing for comparative high-resolution DNA methylation analysis. Nucleic Acids Res 33:5868-5877

17. Joris M, Schloesser M, Baurain D et al (2017) Number of inadvertent RNA targets for morpholino knockdown in Danio rerio is largely underestimated: evidence from the study of Ser/Arg-rich splicing factors. Nucleic Acids Res 45:9547-9557

18. Kok FO, Shin M, Ni CW et al (2015) Reverse genetic screening reveals poor correlation between morpholino-induced and mutant phenotypes in zebrafish. Dev Cell 32:97-108

19. Clark SJ, Harrison J, Paul CL et al (1994) High sensitivity mapping of methylated cytosines. Nucleic Acids Res 22:2990-2997

20. Huang Y, Pastor WA, Shen Y et al (2010) The behaviour of 5-Hydroxymethylcytosine in bisulfite sequencing. PLoS One 5:e8888

21. Moreno-Mateos MA, Vejnar CE, Beaudoin J-D et al (2015) CRISPRscan: designing highly efficient sgRNAs for CRISPR-Cas9 targeting in vivo. Nat Methods 12:982-988

22. Doench JG, Hartenian E, Graham DB et al (2014) Rational design of highly active sgRNAs for CRISPR-Cas9-mediated gene inactivation. Nat Biotechnol 32:1262-1267

23. Li H, Handsaker B, Wysoker A et al (2009) The sequence alignment/map format and SAMtools. Bioinformatics (Oxford, England) 25:2078-2079

24. Tarasov A, Vilella AJ, Cuppen E et al (2015) Sambamba: fast processing of NGS alignment formats. Bioinformatics (Oxford, England) 31:2032-2034

25. de la Rica L, Deniz O, Cheng KC et al (2016) TET-dependent regulation of retrotransposable elements in mouse embryonic stem cells. Genome Biol 17:234

26. Quinlan AR, Hall IM (2010) BEDTools: a flexible suite of utilities for comparing genomic features. Bioinformatics (Oxford, England) 26:841-842

27. Robinson JT, Thorvaldsdóttir H, Winckler W et al (2011) Integrative genomics viewer. Nat Biotechnol 29:24-26

28. Pinello L, Canver MC, Hoban MD et al (2016) Analyzing CRISPR genome-editing experiments with CRISPResso. Nat Biotechnol 34:695-697

29. Wu RS, Lam II, Clay $\mathrm{H}$ et al (2018) A rapid method for directed gene knockout for screening in G0 Zebrafish. Develop Cell 46:112-125. e114

30. Cock PJA, Fields CJ, Goto N et al (2010) The sanger FASTQ file format for sequences with quality scores, and the Solexa/Illumina FASTQ variants. Nucleic Acids Res 38:1767-1771

31. Ross SE, Angeloni A, Geng FS, de Mendoza A, Bogdanovic O (2020) Developmental remodelling of non-CG methylation at satellite DNA repeats. Nucleic Acids Res 48 (22):12675-12688. https://doi.org/10. $1093 / \mathrm{nar} / \mathrm{gkaal} 135$ 
Open Access This chapter is licensed under the terms of the Creative Commons Attribution 4.0 International License (http://creativecommons.org/licenses/by/4.0/), which permits use, sharing, adaptation, distribution and reproduction in any medium or format, as long as you give appropriate credit to the original author(s) and the source, provide a link to the Creative Commons license and indicate if changes were made.

The images or other third party material in this chapter are included in the chapter's Creative Commons license, unless indicated otherwise in a credit line to the material. If material is not included in the chapter's Creative Commons license and your intended use is not permitted by statutory regulation or exceeds the permitted use, you will need to obtain permission directly from the copyright holder. 